\title{
Review Article \\ Stimulated Brillouin Review: Invented 50 Years Ago and Applied Today
}

\author{
Elsa Garmire (i) \\ Thayer School of Engineering, Dartmouth College, Hanover, NH 03755, USA \\ Correspondence should be addressed to Elsa Garmire; garmire@dartmouth.edu
}

Received 1 July 2018; Accepted 3 October 2018; Published 2 December 2018

Academic Editor: Gang-Ding Peng

Copyright (c) 2018 Elsa Garmire. This is an open access article distributed under the Creative Commons Attribution License, which permits unrestricted use, distribution, and reproduction in any medium, provided the original work is properly cited.

\begin{abstract}
Stimulated Brillouin scattering (SBS) is embedded today in a variety of optical systems, such as advanced high-power lasers, sensors, microwave signal processors, scientific instrumentation, and optomechanical systems. Reduction in SBS power requirements involves use of optical fibers, integrated optics, micro-optic devices, and now nano-optics, often in high Q cavities. It has taken fifty years from its earliest invention by conceptual discovery until today for SBS to become a practical and useful technology in a variety of applications. Some of these applications are explained and it is shown how they are tied to particular attributes of SBS: phase conjugation, frequency shifts, low noise, narrow linewidth, frequency combs, optical and microwave signal processing, etc.
\end{abstract}

\section{Conceptual Invention}

As a graduate student at MIT, I was fortunate to have Charles Townes, originator of the maser and laser, as my Ph.D. advisor. In 1963 we published a paper that first introduced the concept that the newly discovered stimulated Raman scattering (SRS) process creates coherent molecular oscillations. Because the incident light in this laser-stimulated process is coherent, the resulting emission can occur by a parametric process due to interaction between the coherent molecular oscillations and the coherent light [1].

When Professor Townes learned that solid state physicists called these oscillations optical phonons, he realized that similar parametric interactions could occur between laser light and the acoustic branch of the phonon spectrum, that is, between laser light and acoustic oscillations, or sound waves. Weak spontaneous Brillouin scattering from acoustic waves had been predicted by Brillouin and already observed using narrow-line spectra. We called this new process stimulated Brillouin scattering (SBS) and set about experiments to demonstrate it.

Our first paper statement of the idea said: "The generation of molecular oscillations or phonons by the interaction between intense maser beams and matter. .. should also lead to generation of intense high-frequency sound waves" [2].
Comparing spectra, the Brillouin shift is much smaller than the Raman shift because the velocity of acoustic waves is much less than the velocity of light. This was already known from the spectrum of spontaneous scattering, where the Raman process gives a much larger shift than Brillouin scattering.

Parametric processes require conservation of both energy and momentum. The pump light suffers an inelastic collision and some of its photon energy is transferred to the acoustic wave; energy conservation requires $\omega_{\mathrm{L}}=\omega_{\mathrm{S}}+\omega_{\mathrm{ac}}$, where $\omega_{\mathrm{L}}$ is the pump laser frequency, $\omega_{S}$ the frequency of the downshifted scattered light, and $\omega_{\mathrm{ac}}$ the frequency of the acoustic wave. The inelastic scattering process robs the incident photons of energy equal to the acoustic vibrational energy, so the scattered photons will be decreased in energy by this amount. This scattered wave, at frequency $\omega_{\mathrm{S}}=\omega_{\mathrm{L}}-\omega_{\mathrm{ac}}$, is traditionally called the Stokes wave.

Conservation of momentum determines the frequency shift in the acoustic wave through a vector equation requiring the momentum before the collision to equal the total momentum after the collision: $\mathbf{k}_{\mathrm{L}}=\mathbf{k}_{\mathrm{S}}+\mathbf{k}_{\mathrm{ac}}$, where the bold print represents vectors. Since the acoustic wave velocity is typically $10^{-5}$ of the velocity of light, the acoustic wave vector will typically be much larger than light's wave vector. The largest $\mathbf{k}_{\mathrm{ac}}$ can and still conserves momentum when the Stokes wave 
is exactly in the backward direction. Then the magnitudes obey $\mathrm{k}_{\mathrm{ac}}=\mathrm{k}_{\mathrm{L}}+\mathrm{k}_{\mathrm{S}}$. Inserting the equality of the wave vector to frequency divided by wave velocity and then simplifying the equation gives $\omega_{\mathrm{ac}}\left(1-\mathrm{v}_{\mathrm{ac}} / \mathrm{v}_{\mathrm{L}}\right)=2 \omega_{\mathrm{L}}\left(\mathrm{v}_{\mathrm{ac}} / \mathrm{v}_{\mathrm{L}}\right)$. Since $\mathrm{v}_{\mathrm{ac}} / \mathrm{v}_{\mathrm{L}}<<$ 1 , a simple form for the Brillouin shift is

$$
\omega_{\mathrm{ac}}=2 \omega_{\mathrm{L}}\left(\frac{\mathrm{v}_{\mathrm{ac}}}{\mathrm{v}_{\mathrm{L}}}\right)
$$

Stimulated Brillouin scattering occurs when a beam of laser light generates a parametric process that simultaneously produces an exactly retroreflected Stokes beam and an acoustic wave traveling in the forward direction. Energy conservation requires that the Stokes beam frequency is reduced from the laser frequency by the frequency of the acoustic wave. Momentum conservation determines that the acoustic wave frequency shift is proportional to the ratio of the acoustic wave velocity to the velocity of the laser beam.

The Brillouin-induced acoustic frequencies are usually very much larger than typical acoustic waves, often called hypersonic. Typical materials are quite lossy at hypersonic frequencies, so hypersonic wave signals decay rapidly in time, causing a broadening to the scattered lines which is proportional to the loss. High resolution spectroscopy of the Brillouin signals can provide considerable information about the high-frequency mechanical elasticity of these materials.

Any narrow-line light source sees spontaneous Brillouin as weak inelastic scattering from thermal acoustic waves. When the waves are moving away from the light beam, the largest signal is observed in retroreflection and the Stokes shift moves to longer wavelengths, as in stimulated Raman scattering. When the acoustic waves are moving toward the light beam, their frequency will add to the frequency of the laser beam and the scattered light will have a frequency increased by the acoustic wave frequency. This is called the anti-Stokes wave.

Historically the term "Stokes" was named for Sir George Gabriel Stokes, who in 1852 described the change in wavelength of fluorescence, which is always at lower photon energy than the incident light. When Raman scattering was discovered, similar shift to lower photon energy was called Stokes light. When Raman scattering was discovered to have weak signals at shorter wavelength than the incident light, this was called "anti-Stokes" light. The terminology has stuck and is used in SBS as well.

\section{Initial Experimental Demonstrations and Theory}

The first experimental demonstration of SBS was in solids, by Chiao, Townes, and Stoicheff [3]. Chiao was a graduate student in our group who joined after I did and Stoicheff was a visitor from Canada who was an expert in FabryPerot interferometers. He taught us how to use this etalon to separate very small frequency shifts. The Brillouin Stokes line showed up in interferograms with smaller frequencies (at smaller radii of curvature) than the laser line, as shown in Figure 1. The results validated our theory.

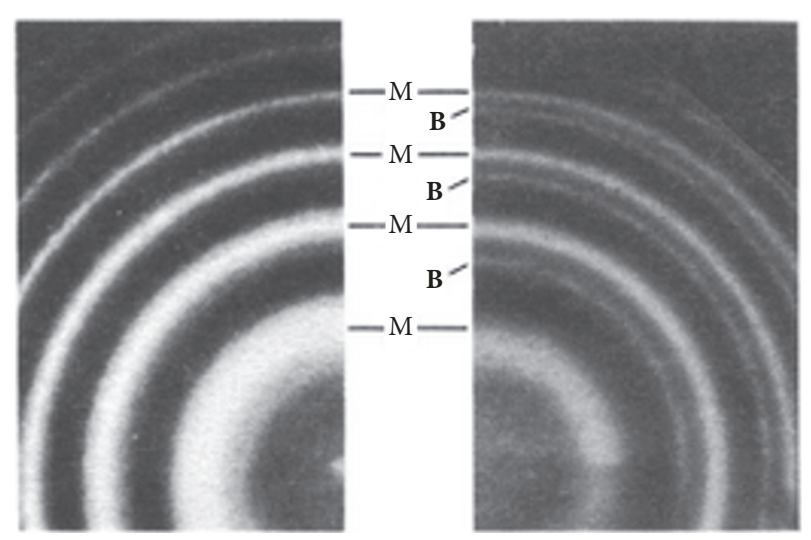

FIGURE 1: Fabry-Perot interferograms of Q-switched ruby laser radiation (rings labeled $\mathrm{M}$ ) and of the Brillouin scattered Stokes radiation (rings labeled B). Left shows laser alone, right is observation with the laser focused into quartz [adapted from [3]].

At this time I had been experimenting on stimulated Raman scattering (SRS) in liquids pumped by a ruby laser and was puzzling over a strange result when I focused the ruby laser beam into a $10 \mathrm{~cm}$ long cell of liquid. The incident laser light was monitored by reflecting out a small fraction of the light onto a photodetector by means of an angled glass plate. An opaque screen in front of the liquid cell made sure only laser light was being measured. Removing the opaque screen, I was surprised to find that now the power coming from the laser increased by a factor of 10 . Why was this happening? Raman scattering was not known to produce a reflected signal, and I made sure the cell was canted at an angle, so as to eliminate reflected laser light. This all happened a year before we predicted that SBS should cause retroreflection.

After we invented the concept of SBS, I inserted a FabryPerot into the optical path, as in [3], and immediately observed SBS right away (see Figure 2), but I did not at first understand what I was observing. Theory said there should be only one Brillouin shift (assuming no external feedback), but I observed several orders of Brillouin shift-up to six in some cases. After studying this conundrum, I concluded that the backward Brillouin Stokes reentered the laser. My new idea was that because of the Stokes frequency shift, this Brillouin Stokes line could experience unsaturated gain in the inhomogeneously broadened ruby laser. So the Stokes beam, re-reflected by the back laser mirror, returned to the liquid as a strong signal, joining the initial laser beam as a new wavelength, strong enough to cause its own Brillouin shift. This led to the unexpected Stokes cascade observed in the data-all due to gain in the ruby laser [4].

With these experimental successes, we submitted a patent for SBS in 1964. The patent explained SBS as a parametric amplifier: "Two mechanisms amplify wave energy by stimulated emission: (1) Creating population inversion: the laser; (2) Pumping coherent energy into a medium through which wave energy propagates: the parametric amplifier" [8].

"The Parametric amplifier does not require population inversion. Coherent pumping energy drives the system coherently and phase relations that are set up in the medium 

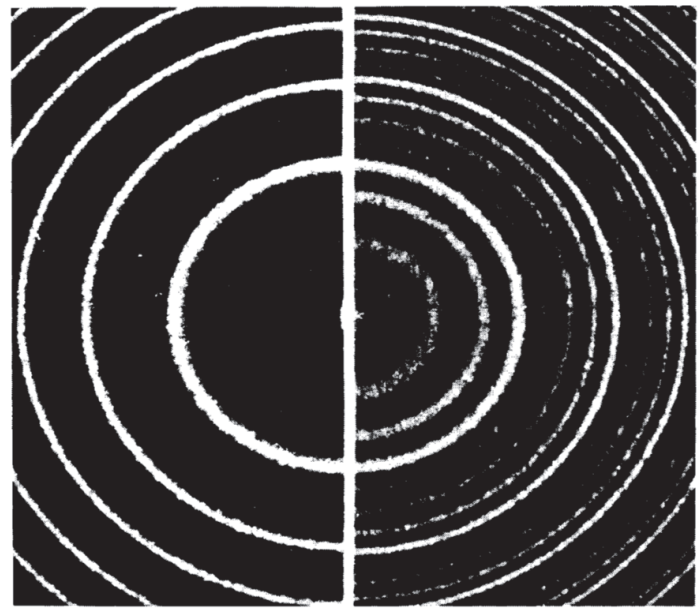

FIgURE 2: Fabry-Perot interferogram from Q-switched ruby laser (left) and from SBS in nitrobenzene (right). After the first order Stokes ring, additional rings are attributed to phase conjugation returning the Stokes light to the laser where it experiences additional gain in the inhomogeneously broadened ruby laser [adapted from [4]].

allow amplification even without population inversion.... A nonlinear medium can amplify a wave of another frequency." We applied this concept to acoustic waves. Our claims were essentially:

(i) "Means for generating and amplifying acoustic wave energy in a medium by directing EM radiation to the medium.

(ii) Means for generating and amplifying acoustic energy in a medium in response to incident EM radiation so that substantial EM radiation of another frequency is generated in the medium.

(iii) A tunable source of intense coherent EM radiation.

(iv) Means for broadening envelope of laser frequencies

(v) Generating EM radiation from material or acoustic waves.

(vi) Combining two coherent beams of EM radiation to produce relatively intense acoustic waves in medium.

(vii) Providing a resonant cavity to lower the required threshold power and providing feedback."

Almost all of these concepts have proven successful, but time ran out on the patent to make us rich; it took more than 30 years before developments looked promising!

\section{Parametric Basis of the SBS}

SBS occurs in any medium (liquid, solids, gases, plasmas) by interaction of laser light and a coherent density grating caused primarily (but not exclusively) by electrostriction.

The laser reflects off the grating, which moves with velocity $\mathrm{v}_{\mathrm{ac}}$; it will be Doppler-shifted, and scattered light will experience a wavelength-shift in a coherent inelastic scattering process. Electrostriction is typically a small nonlinearity; practical use typically requires Q-switched lasers, long lengths of fibers, or high $\mathrm{Q}$ resonators. The basic concept is the classical parametric amplifier. The interaction between two coherent waves (the pump and the acoustic wave idler) provides amplification to a Stokes wave (the signal), which experiences a gain per unit length. In real systems, this gain must be offset by any loss per unit length in the medium. As with any parametric amplifier, the gain builds from whatever input signal is present. In most cases there is no input signal, except for noise; spontaneous Stokes waves occur by scattering from thermally excited acoustic vibrations.

The critical power for observing SBS when there is no input signal has conventionally been defined as 21 times the power required to reach transparency for the transmitted signal. Given that the input is just a few acoustic phonons, the factor of 21 is traditionally required to provide enough gain for an easily measurable signal. This critical power is given by [5]

$$
P_{L}^{\text {crit }}(0)=21 \frac{A_{e f f}}{g_{9} L_{e f f}}
$$

where $A_{e f f}$ is the effective area of the beam, $L_{e f f}$ is the effective length of the gain region, and $g_{o}$ is the peak gain coefficient. This simple equation consists of geometrical factors and lumps everything else into a peak gain coefficient. The peak gain coefficient $g_{o}$, shown below, [5] contains all the physical parameters of the Brillouin material, with $\gamma_{e}$ as the electrostriction coefficient, which serves as the source of the nonlinearity. Here $n$ is refractive index, $v_{a c}$ is the velocity of acoustic phonons, $\rho$ is the average medium density, and $\Gamma_{\mathrm{B}}$ is the Brillouin linewidth angular frequency. The gain $g$ falls off from its peak value at $\Omega_{\mathrm{B}}$ by the typical Lorentzian lineshape:

$$
\begin{aligned}
& g_{o}=\frac{\omega_{s}^{2} \gamma_{e}^{2}}{c^{3} n v_{a c} \rho \Gamma_{B}}, \\
& g=g_{o} \frac{\left(\Gamma_{\mathrm{B}} / 2\right)^{2}}{\left(\Omega_{\mathrm{B}}-\Omega\right)^{2}+\left(\Gamma_{\mathrm{B}} / 2\right)^{2}}
\end{aligned}
$$

Notice that the equation for the gain coefficient does not contain Planck's constant $h$. This means the SBS process can be understood completely classically. This is expected because acoustic waves are not affected by quantum mechanics. The noise that begins the SBS process is found by thermodynamics, and not by quantum mechanics.

The Lorentzian linewidth factor becomes important when the laser pump is narrow-line and the Brillouin linewidth narrows. In many cases, however, the SBS spectrum has a broad-enough linewidth that it is sufficient to use the peak gain coefficient when calculating the Brillouin gain.

To give an idea of experimental numbers, for Corning fiber SMF-28, the critical power at threshold has been measured with a narrow-line DFB laser as approximately $4 \mathrm{~mW}$. Brillouin shifts are $\sim 10 \mathrm{GHz}$ and linewidths are $\sim 20 \mathrm{MHz}$.

As the laser increases in power beyond the critical power, the Stokes output increases exponentially in the backward direction. As the incident laser light is depleted, the Stokes 
power will saturate to a level that is comparable to the incident pump.

If the acoustic wave is moving away from the incident laser beam, the Doppler shift means that the reflected Stokes wave must be red-shifted from the incident light. Forward blue-shifted anti-Stokes waves may occur from acoustic waves traveling in the same direction as the pump. If two pumps of equal intensity enter the medium, one from each side, the two moving gratings will interfere to become an oscillating stationary wave. This is called a Brillouin dynamic grating, a SBS-created stationary, oscillating density wave in the material that can diffract a separate light beam (e.g., at a different polarization), with its own interesting applications.

\section{Characterizing SBS Applications}

Now we want to look at applications of SBS. The Web of Science shows 4875 archival research papers on SBS until now. There is no way all these papers can be reviewed, nor even all the applications that these publications cite. How do we review applications that are almost innumerable? How do we characterize these applications? I choose to consider applications in terms of the optical parameters by which the SBS process interacts with the medium. These include the following:

(i) Spatial dependence: Phase-conjugate reflection; beam cleanup; beam combining

(ii) Time dependence: Pulse shortening, reshaping; increasing self-phase modulation

(iii) Frequency dependence: Sensors; spectroscopy; sensors of strain and temperature

(iv) Linewidth dependence: Optical filters, frequency combs

(v) Background noise: Low noise amplifiers, lasers and microwave generators

(vi) The physical hypersonic waves: Brillouin dynamic gratings, sensors

(vii) Amplitude of SBS signal: Optical signal processing

(viii) Micro-devices: Frequency combs, low critical power devices

(ix) Nanostructures: Opening up new ways of thinking about light-materials interactions.

Each of these will be explored in the following sections. No attempt is given to trace the development of each application. The work referred to here is chosen to be clearly illustrative of the SBS mechanism for the application. The papers will not be the first, nor the last, in that field. For further research on any given application, the author recommends a literature search using the application as one of the key words.

\section{Specific $21^{\text {st }}$ Century Applications of Stimulated Brillouin Scattering}

5.1. Applications in the Spatial Domain. Optical phase conjugation is the physical phenomenon that will be described in this section. It can control the spatial phase front of a coherent beam and is also used in beam combining, where the relative phase is crucial.

5.2. Phase Conjugation. In 1972 Boris Zeldovich showed theoretically and experimentally that a phase-conjugate mirror could compensate for distortion in a laser beam [15]. SBS provided him a reflected output beam with a phase front as planar as the input laser beam. This is specifically the result of the $\pi$ phase shift upon SBS reflection, a phenomenon not seen from ordinary mirrors [15].

When light reflects from a conventional mirror, its phase continues to evolve, as if the mirror were not there. (That is why an ordinary mirror projects us into virtual space.) In contrast, the beam reflected by an SBS phase-conjugate mirror (PCM) has its phase "conjugated." That is, the sign of the phase reverses. Thus the beam appears to propagate exactly backward and the negative sign of the reversed phase cancels all distortions the wave experiences when traveling toward the mirror. Assuming the beam started out as diffractionlimited, with a smooth wavefront, any introduced wavefront distortion will be cancelled in the SBS retroreflected beam due to the phase reversal function of SBS at the PCM. The initial diffraction-limited wave will be fully recovered in the Stokes wave, as the initial distortion introduced on the laser beam "unwinds" on the Stokes beam as it passes back through the distortion, as shown in Figure 3.

Having realized this, I immediately saw an error in my 1964 SBS paper [4]. I had shown an off-axis Stokes beams reflecting at an angle equal to the incidence angle, as with an ordinary mirror. This is not true SBS retroreflection, as Zeldovich pointed out, which requires phase conjugation. This concept immediately explained why my lens and other optical elements did not ruin the retroreflected Stokes beam and allowed it to be a well-behaved beam as it entered and traveled through the ruby laser, receiving gain.

This also answered the question I had had since 1963, when I observed that more light came out of the laser when SRS was taking present. Now I understood that the extra light was Stokes from SBS that accompanied the SRS; high quality phase conjugation ensured that my assumed concept of gain in the ruby made sense from an optical point of view.

The lesson to be learned here is that what is not understood in an experiment can eventually be explained, with enough research, although in this case it took almost 10 years.

After Zeldovich's initial report, researchers investigated laser bean cleanup from phase-conjugate mirrors (PCM) using SBS. Experiments showed the effect dramatically, as shown in Figure 4. Figure 4(a) shows fluctuations in a YAG laser beam occurring due to nonuniform phase distortion from imperfect components and localized thermal heating from flash lamps. Figure 4(b) shows the YAG Stokes beam when the ordinary mirror was replaced by an SBS PCM; the perfection of the Gaussian beam is impressive. The Brillouin PCM, used as the back-end mirror of the YAG laser, contained a lens and a cell of acetone [6].

Such SBS beam cleanup is often used in fiber lasers. In MOPA configurations, multimode fibers can provide powerful amplifiers, without damage to the fiber. As long as 


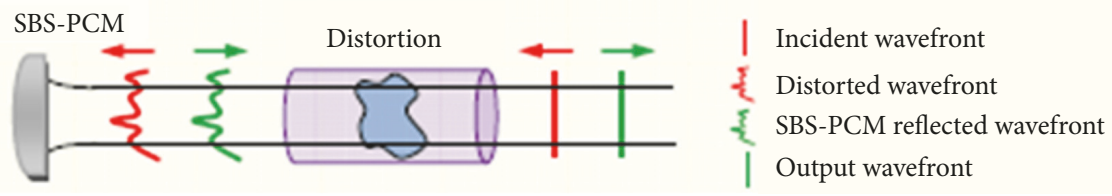

FIGURE 3: Distortion compensation with a SBS-phase-conjugate mirror [adapted from [5]].

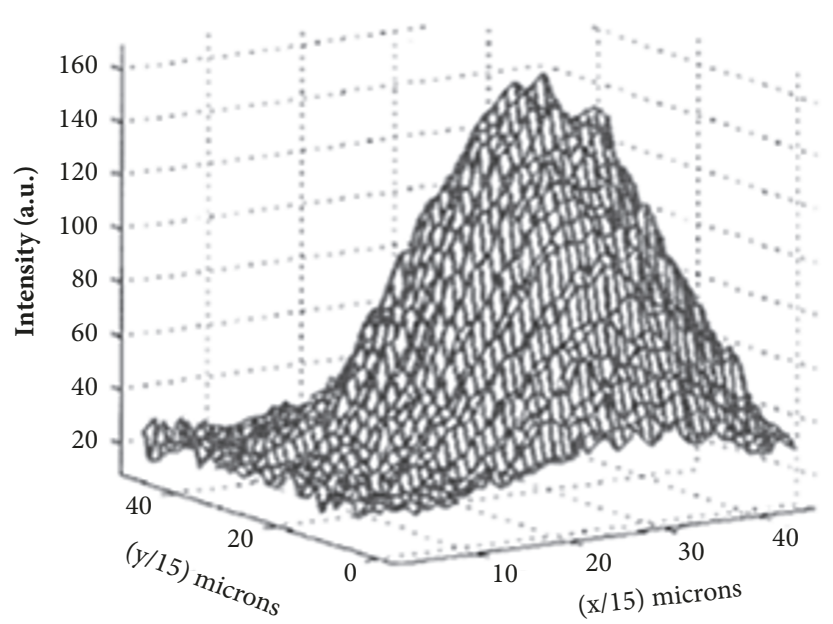

(a)

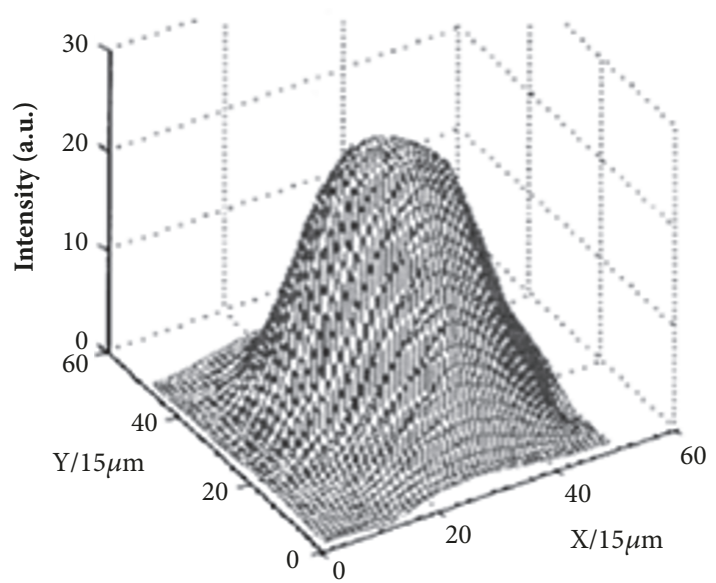

(b)

FIGURE 4: (a) YAG laser beam experimental spatial dependence, showing a myriad of distortions; (b) experimental results with a phaseconjugate mirror, showing beam cleanup to a 2D Gaussian profile [adapted from [6]].

the input is a high quality Gaussian beam, providing a PCM as the last element of the chain unwinds all distortions on the Stokes and it will come out diffraction-limited. High-power lasers now often depend on SBS beam cleanup.

PCM Multiple Beam Combining is another important application of phase conjugation. One example is a project underway in South Korea, using SBS PCMs to combine the output of four high-power laser amplifiers into one coherent beam. This combined laser system is designed to provide an average power of $4 \mathrm{~kW}$ in a series of nanosecond pulses. The master oscillator-power amplifier configuration has a front end that produces an average of 10 Watts which a preamp increases to $120 \mathrm{~W}$. This single beam is then divided into 4 beams, each traveling through a double-pass amplifier to a power of $1 \mathrm{~kW}$ per amplifier. If SBS phase-conjugate mirrors are used in each amplifier, the resulting Stokes beams can, in principle, unwind any phase differences between each amplifier optical path. This should enable the smooth wave front of the initial master oscillator to be created when these beams are combined coherently, because of the self-locking SBS PC mirrors. As of this writing, these researchers are still looking for the most practical beam combiner [19]. The method has an exciting potential, but is quite complex.

5.3. Brillouin Microscopy and Imaging. Brillouin microscopy was introduced in 2007 [7], driven by the interest in cellular and tissue biomechanics and the ability to determine viscoelastic properties of materials on a microscopic scale.
This process uses scanning confocal microscopy and a special VIPA (virtually imaged-phase-array) etalon, designed to be placed in the focal plane at an angle to the laser beam, so successive reflections appear as a phased array. The VIPA converts a single input beam into a series of parallel output beams of gradually decreasing intensity. These beams constructively interfere at an angle that depends on the wavelength. Placing a lens between the VIPA and an array detector (CCD or similar) allows recording the spectrum of the input light. Each subsequent beam has a precise increase in phase and fixed lateral displacement, hence a "phased array." Parallel spectral detection is enabled by collecting the entire Brillouin spectrum in one pulse with sub- $\mathrm{GHz}$ resolution and high throughput efficiency. An additional Fabry-Perot etalon acts as an ultra-narrow band-pass filter for Stokes light. Figure 5 shows one published example of measurements made with such a system. Changes in elasticity were observed during UV-induced crosslinking; distinct phases can be seen, characterized by constant Brillouin shift before curing, dynamic changes during UV crosslinking of polymer under illumination, an increase in the shift after illumination due to crosslinking, and a steady state after being fully cured. The elastic modulus was calculated from the Brillouin shift [7].

Brillouin microscopy has been reported to be effective in a variety of studies, such as the following:

(i) biological applications in cell and subcellular biomechanics 


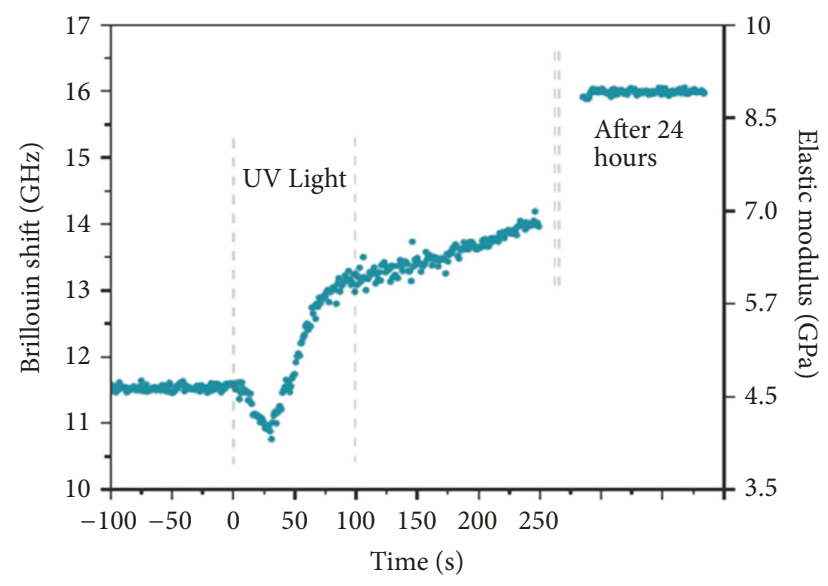

FIGURE 5: Real-time monitoring Brillouin shift frequency during UV-induced crosslinking of polymer [adapted from [7]].

(ii) corneal biomechanics; monitoring changes in crystalline lenses

(iii) determining elastic properties of trabecula bone connectors

(iv) studying selective two-photon collagen crosslinking

(v) quantification of plaque stiffness in blood vessels in order to determine rupture potential

(vi) mechanical properties of a variety of composite materials and interfaces; glass-to-metal-seals, etc.

(vii) assessing diamond-like carbon

(viii) monitoring chemical reactions in soft media (e.g., Figure 10)

(ix) Imaging mechanical or microwave induced phonons in lithium niobate.

Research is continuing to improve the technology, by adding techniques such as adaptive optics [23] and dark field microscopy to compensate for murkiness in biological samples. However, to date these systems generally use spontaneous and not stimulated Brillouin scattering. A different technique starts from creating Brillouin dynamic gratings (BDG) with interfering pulsed laser beams, discussed next.

5.4. Impulsive Stimulated Brillouin Scattering. This technique begins with Brillouin dynamic gratings, which are standing acoustic waves that come from interfering two traveling acoustic waves of equal intensity, which had, themselves, been created by SBS from two interfering laser beams. The Stokes beams are created by splitting an intense laser beam into two beams, each of which creates its own Stokes beam in the material to be studied, as shown in Figure 6. The resulting interfering acoustic waves provide a density gradient modulated at the acoustic frequency. A cw probe laser, focused on this density gradient, will scatter preferentially at the Bragg angle and be modulated at the frequency of the acoustic wave. As a result, a transmitted (or reflected) $\mathrm{cw}$ probe beam will be modulated in intensity whenever the intense, interfering laser beams are present. A simple high-speed photodiode can monitor the modulation frequency and provide a signal that can be heterodyned with the cw probe light to provide a measure of the Brillouin frequency shift and linewidth [9].

The periodicity of the standing acoustic wave is controlled by the intersection angle of the two pump beams while its frequency is picked up by a cw probe beam that scatters from the acoustic wave. The stationary, but dynamic, acoustic grating scatters some of the probe light out of the beam and its transmission will be reduced, as sketched in Figure 6(a). The frequency of this acoustic wave is given by $\Omega_{\mathrm{B}}=2\left(\mathrm{v}_{\mathrm{ac}} / \lambda_{\mathrm{L}}\right) \sin \Theta$, where $\Theta$ is the angle between the intersecting beams measured from outside the material. The scattered probe is modulated at the acoustic frequency, detected with a photodiode and collected by an oscilloscope, Figure 6(b). Each pixel in the scan has a trace that is recorded, an FFT taken (Figure 7), and fitted to find the center frequency with a resolution $\sim 1 \mathrm{MHz}$ and $\mathrm{SNR} \sim 8$. Images are created by scanning in $2 \mathrm{D}$ and plotting the frequency shifts at each pixel.

This impulsive stimulated Brillouin scattering (ISBS) process uses super-intense ps laser pulses that act instantaneously to create super-intense Stokes light and large-amplitude stationary acoustic waves. This provides a large modulation depth on the cw probe and speeds up the measurements. The ISBS process has the advantage of not requiring frequencytuning a laser to a SBS resonance.

5.5. SBS Beating between Tunable Pump and Fixed Probe. Previous to this experiment, the same research group tried experimenting with two counter-propagating $\mathrm{cw}$ semiconductor lasers at $780 \mathrm{~nm}$. A fixed probe laser and a tunable, counter-propagating pump laser were focused inside a sample to generate the SBS signal. The counter-propagating pump and probe beams with center frequencies at $f_{1}$ and $f_{2}$, respectively, beat together in the medium, which generated and amplified the sound wave in the medium which oscillated at the beat frequency $f_{\text {beat }}=f_{1}-f_{2}$ when the beat frequency matched the acoustic frequency of the medium [10]. This technique required one laser frequency to be tunable. Figure 7 shows this system monitoring the transmission spectrum of water by the intensity of transmitted probe light as a function of the frequency of the tunable pump light. Loss and gain peaks at the Brillouin shift frequency are observed on either side of the scattered pump light. The signal on the Stokes side is reduced when the pump-probe frequency difference matches the Brillouin shift by scattering from the pumpinduced SBS acoustic grating. The positive frequency gain (anti-Stokes) occurs due to scattering from already-excited phonons. Others have seen gain on the anti-Stokes side, which requires two oppositely traveling laser beams.

5.6. Stimulated Brillouin Spectroscopy. A group in Israel used the same technique but added lock-in detection by modulating the pump and probe, each with its own frequency. Their desire was to develop a system capable of imaging and needed a rapid way to detect the Brillouin frequency shift. Their aim was to measure transmission SBS spectra with stimulated 


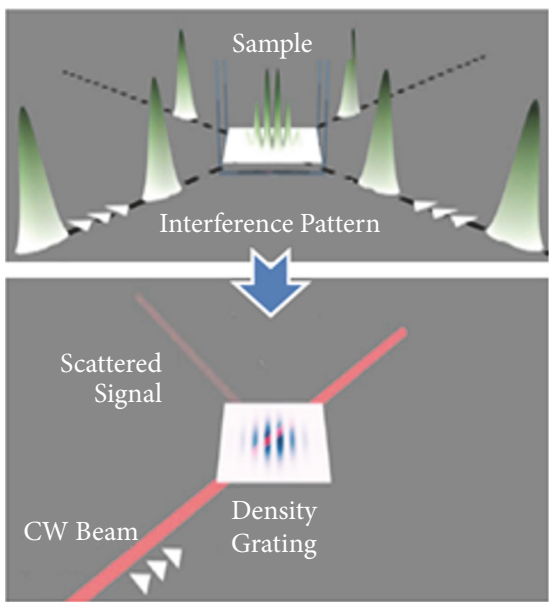

(a)

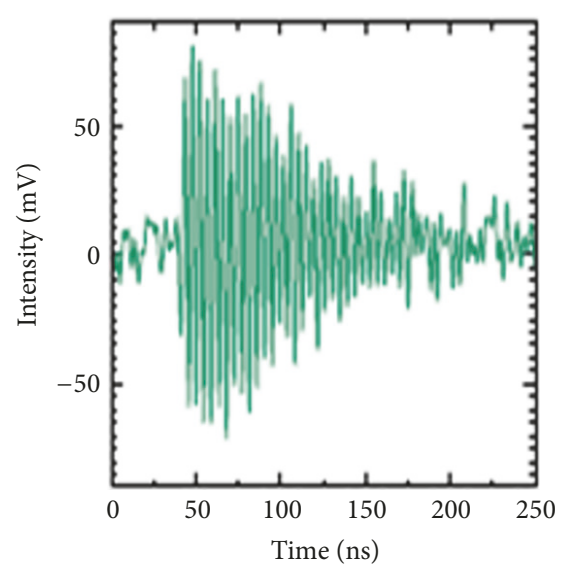

(b)

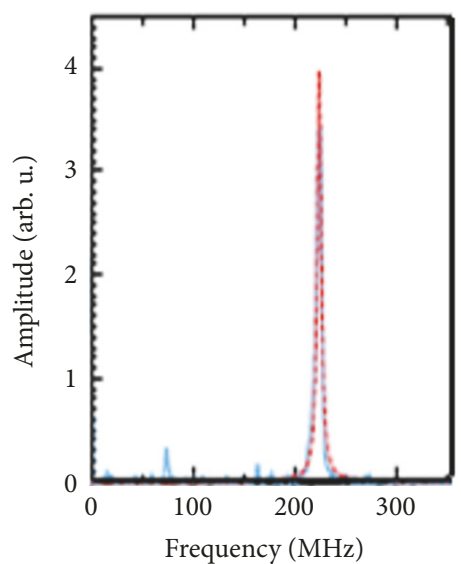

(c)

FIGURE 6: (a) Conceptual sketch of impulsive stimulated Brillouin scattering (ISBS), with psec pulses creating interfering acoustic waves through SBS. This modulates the probe, picked up by a high-speed photodetector, whose signal is shown in (b). The Fourier transform of this transient data is shown in (c), allowing direct determination of the Brillouin frequency shift and linewidth [adapted from [9]].

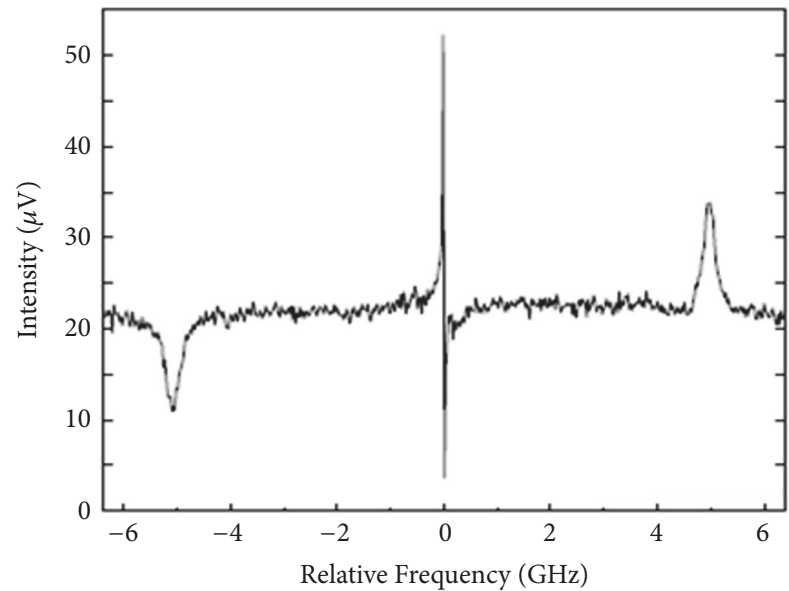

FIGURE 7: SBS spectrum of water, showing intensity of probe versus frequency of pump [adapted from [10]].

gain peak levels as low as $\sim 10^{-6}$ of the pump, good-to-high SNR, while maintaining frequency scanning rates of $20 \mathrm{MHz}$ per $10 \mathrm{~ms}$. They used frequency-domain SBS spectroscopy with standard single-modulation lock-in detection (SM-LID) by adding an ultra-narrowband hot rubidium- 85 notch filter to which the pump wavelength was tuned. The pump beam was modulated by an acoustic-optic modulator and SBS was lock-in detected at that same frequency. The probe was tuned to a wavelength outside the stopband of the filter to obtain maximum SBS signal, and the Brillouin frequency shift was lock-in detected, as in standard single-modulation phasesensitive detection schemes. They demonstrated images at frequency scanning rates 100- and 10-fold faster than other SBS spectroscopy systems [24].

All the experiments mentioned above were motivated by future applications in medicine. However these experiments also point out the reality of creating density waves oscillating at acoustic frequencies in different media for variety of applications. These have been called Brillouin dynamic gratings (BDG) and have found many uses, both in science and in the burgeoning field of optomechanics, as will be discussed later.

5.7. Applications of SBS in the Time Domain. Moving the parameter from space to time, SBS has been shown to provide useful pulse compression. Figure 8(a) shows how the Stokes pulse moving backward through an input pulse increases in steepness. Experimental results have shown that SBS can shorten a $7.8 \mathrm{nsec}$ pump pulse to $450 \mathrm{psec}$, a factor of 17, with results shown in Figure 8(b) [11]. The Nd:YAG oscillator, mode-locked by a Cr:YAG crystal, produced $7 \mathrm{~ns}$ pulses. The SBS pulse compressor consisted of a flash-lamppumped Nd:YAG amplifier and a SBS cell Stokes generating cell. The SBS medium was a multiconstituent perfluorinated compound called FC-770. The SBS pulse compressor was designed with a phase-conjugate mirror, which compensated for distortions built up in the YAG amplifier, yielding a high quality single mode profile.

The shortest pulses are compressed from 9 ns down to $170 \mathrm{ps}$, a compression factor of 53 times. However, this only occurs when the system is optimized for optical power, given an initial pulse length and a specific Brillouin nonlinear coefficient. The effect of time-narrowing is comparable to the effect of gain-narrowing [25].

5.8. Applications of SBS in the Frequency Domain. Measuring the Brillouin frequency shift has been shown to be a very useful way for optical sensing applications. The Brillouin frequency shifts from the frequency if the input laser in standard telecommunication fibers is $10.74 \mathrm{GHz}$. The shape is Lorentzian, with a linewidth of $20 \mathrm{MHz}$. These frequencies can be measured by heterodyning the long-wave-shifted Stokes with the laser pump. 


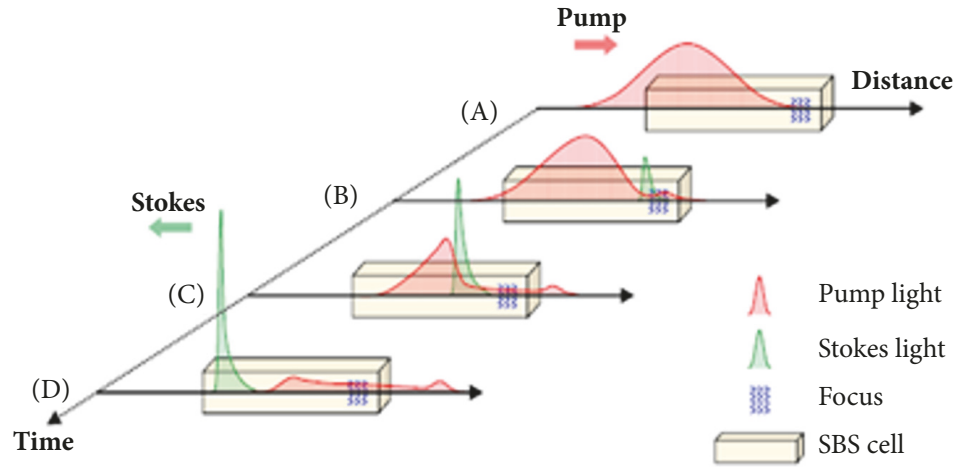

(a)

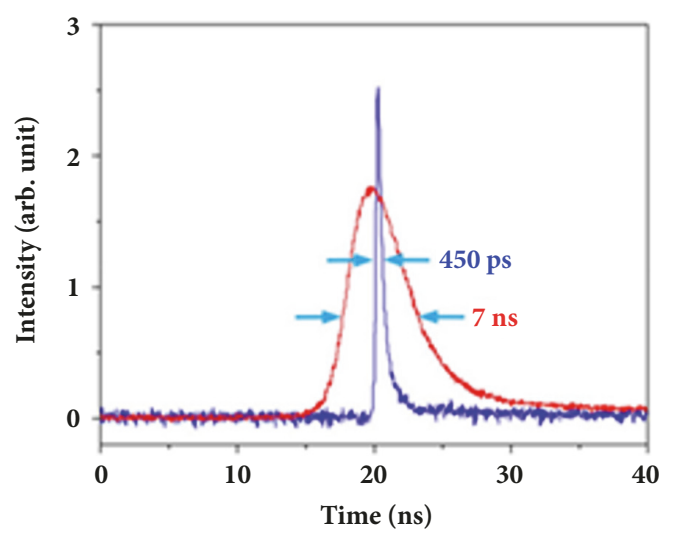

(b)

FiguRE 8: (a) Schematic of short pulse formation; (b) some experimental results by the University of New Mexico group [adapted from [11]].

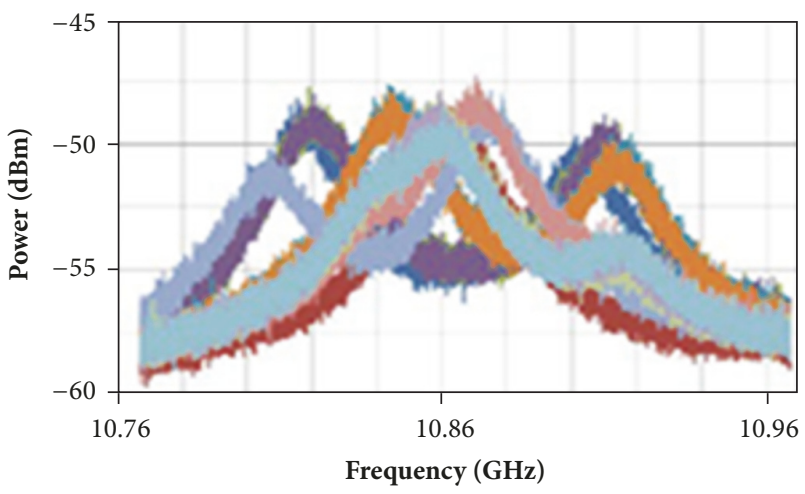

FIGURE 9: High resolution heterodyne measurement of SBS shift in several lengths of the same (Corning SMF-28) single mode fiber [adapted from [12]].

The use of SBS in fibers as a sensor comes from the fact that the Brillouin frequency shift depends strongly on the local refractive index. The Brillouin shift can be different for different fibers; even fibers from the same manufactured lot can have different shifts, as seen in Figure 9. This data was obtained by cutting an initial fiber into similar discrete lengths and comparing their Brillouin shifts. Measurements were made by heterodyning the Stokes signal with the initial laser pump on the surface of a high-speed detector [12]. The variations in Brillouin shift for this particular fiber denote a variation in refractive index sufficient to show different Stokes shifts. The sensitivity of the Brillouin shift to the local refractive index depends on the materials in the core and cladding and the size of the core. Anything that varies the frequency shift can be sensed by measuring changes in the frequency of the Brillouin shift. Traditionally the largest effects are temperature and strain.

5.9. Brillouin Fiber Sensors. Understanding the importance of Brillouin fiber sensors begins with understanding optical time delay reflectometry (OTDR). Measurements of time delay begin by focusing a train of laser pulses into an optical fiber. These pulses travel some distance down the fiber, and after the time delay it takes the light to get there, it senses a perturbation in the fiber's refractive index. Typically this perturbation reflects a bit of the signal back to the origin. The time delay between excitation and reflection, measured at the input end, will represent twice the distance to the perturbation. Measuring the time delay determines the location of the perturbation, after converting units with the velocity of light.

Spontaneous Brillouin scattering can provide a much more sensitive fiber sensor than traditional OTDR because it continuously scatters some light back to the origin as Stokes light, which is directly sensitive to perturbations in the fiber. Brillouin OTDR (or BOTDR) is observed by optical filtering to monitor signal only at the Stokes frequency. Heterodyning the return signal with the incident pump returns the Brillouin frequency shift, around $10 \mathrm{GHz}$.

Converting spontaneous BOTDR systems to stimulated Brillouin scattering provides a narrower linewidth Stokes signal, with more power, all of which offer higher accuracy and sensitivity. With SBS, Brillouin sensing has been reported to distances over $100 \mathrm{~km}$ in length.

A conundrum remains, however, with these sensors. A single frequency shift cannot separate dependence on strain from dependence on temperature. Solving this puzzle creates a more useful Brillouin sensor. A variety of system designs have been suggested to overcome this issue. Only one method is presented here: a multicore fiber that demonstrates different acoustic frequencies in the central core from cores on the outer edge. After fabrication, the measured thermal shift was shown to be different for the core in the center and that on the edge, as shown in Figure 10, allowing temperature effects to be separated from strain [13].

The term Brillouin Optical Time Domain Analysis or BOTDA has been introduced for these sensors, which have, without a doubt, become the greatest commercial success of SBS. A number of approaches to improving the properties of BOTDA systems have been suggested. My literature search of Brillouin sensors on Web of Science found 1,701 papers since 1989. Clearly further understanding of these sensors is a field in itself! 


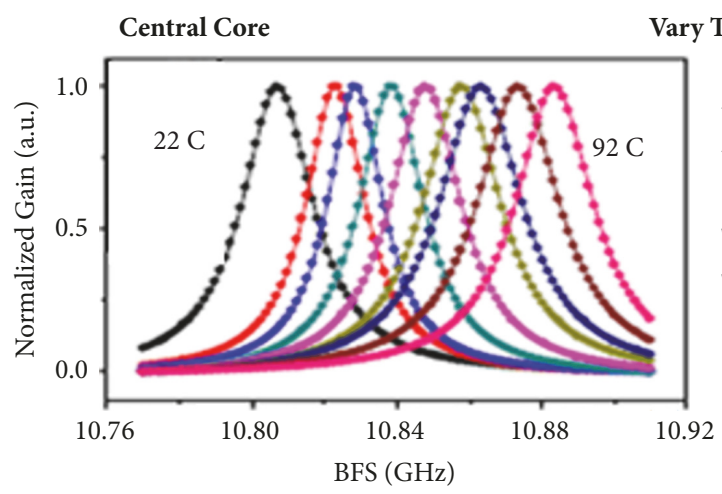

(a)

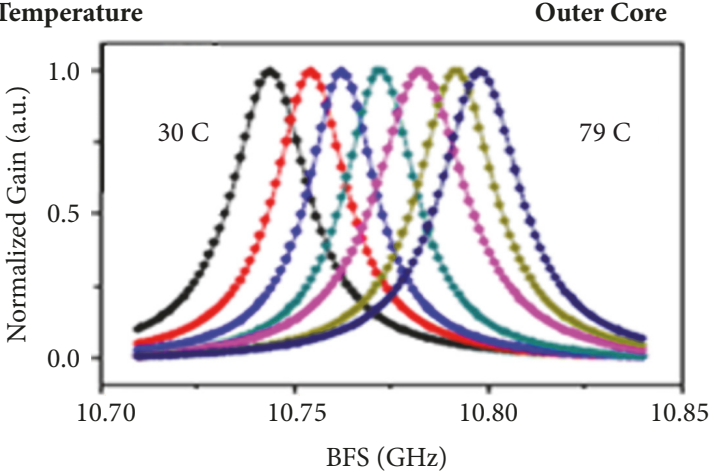

(b)

FIGURE 10: Comparison of SBS frequency shift as a function of temperature in a multicore fiber, comparing the difference between the central and an outer core [adapted from [13]].

Several advances should be noted, but will not be discussed here:

(i) Spatial resolution of $8.3 \mathrm{~mm}$ demonstrated over $17.5 \mathrm{~km}$ distance, using "correlation-domain analysis combined with temporal gating of pump and time domain acquisition." At this point there can be more than $1,000,000$ resolvable points.

(ii) Wide strain-range dynamic measurements use "slope-assisted BOTDA," based on vector SBS and frequency-agile techniques, or by using Brillouin Phase-Gain ratio [26].

(iii) Coherent BOTDA using phase- and polarizationdiversity and heterodyne detection and embedded digital signal processing [27].

(iv) Double-pulse measurements; polarization effects, total Distance $>100 \mathrm{~km}$, other parameters: magnetic field, vibration, etc. [28].

Fiber sensors are embedded into numerous buildings, bridges, along pipes, and many other civil engineering projects. They warn of earthquakes, weakening structures, intrusion of bad guys, etc. Their usefulness is limited only by the creativity of individual engineers!

Brillouin scattering for imaging sensors was discussed under spatial applications. This is a reminder here that Brillouin sensing is used in microscopy, to filter or monitor $2 \mathrm{D}$ image locations where SBS is particularly strong. Interfering the laser pump light and returned SBS light can provide a heterodyne measurement of the frequency shift, which makes it possible to compare images, monitoring different mechanical properties. This is particularly important for biology, biomedicine, and studies of other spatially varying soft matter.

5.10. Disadvantages of SBS. It is important to point out that not only SBS makes major contributions to technology, but can also make major detributions to technology. I've hereby invented a new word: "detribution." We all know what a contribution is; a detribution is the opposite. As an example, sometimes a paper is a detribution. When it is published, the body of knowledge is decreased rather than increased. Publishing incorrect data, or claiming data that never existed, can be a detribution to scientific literature.

The detribution that SBS makes is to limit the power transmitted through optical fibers; if the input laser exceeds a certain critical SBS power, a fraction of the input power is converted to backward-traveling Stokes reflection. This detribution process was realized as early as 1972; it saturates the optical power that can be transmitted in an optical fiber system. In addition, scattering from the backward signal can introduce noise in a transmitted signal. It has been crucial for telecommunications technology to overcome SBS in fibers. There is a similar need to overcome stimulated Raman scattering in fibers, but this does not occur until the existence of much higher critical powers than SBS. The SBS process dominates in telecommunication fibers as the power is increased beyond the $4 \mathrm{~mW}$ critical power.

The most effective method to suppress SBS is typically to broaden the laser linewidth. This works very effectively to increase SBS threshold, since the Lorentzian peak intensity decreases, as shown in the gain-linewidth equation. The broadening is usually carried out by frequency-modulation of a narrow pump laser line. Continuous wave lasers with very narrow linewidth (e.g., $<10 \mathrm{MHz}$ wide) have SBS threshold power of a few $\mathrm{mW}$ in fibers at telecom wavelengths. The formula for the gain coefficient in (3) shows that threshold depends on the inverse of its spectral linewidth. Engineers have found a variety of ways to modulate distributed feedback lasers in order to broaden their spectrum.

A look at the gain formula implies other possible approaches to raising the Brillouin threshold and decreasing its detribution to communication systems. Limiting fiber length raises the critical power. If a longer length is required, SBS can be suppressed by concatenating fiber cables, each with a different Brillouin shift. Other methods include varying the fiber along its length to change the Brillouin shift with distance. This variation could be in core size, temperature, or even strain. 


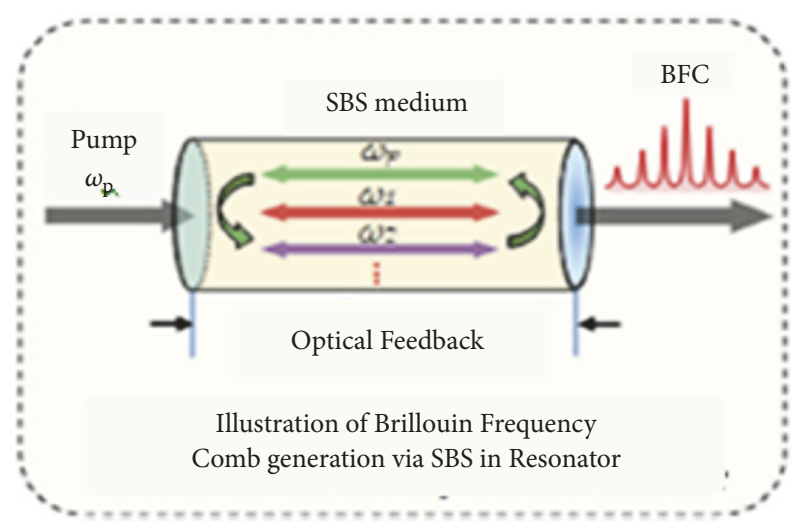

(a)

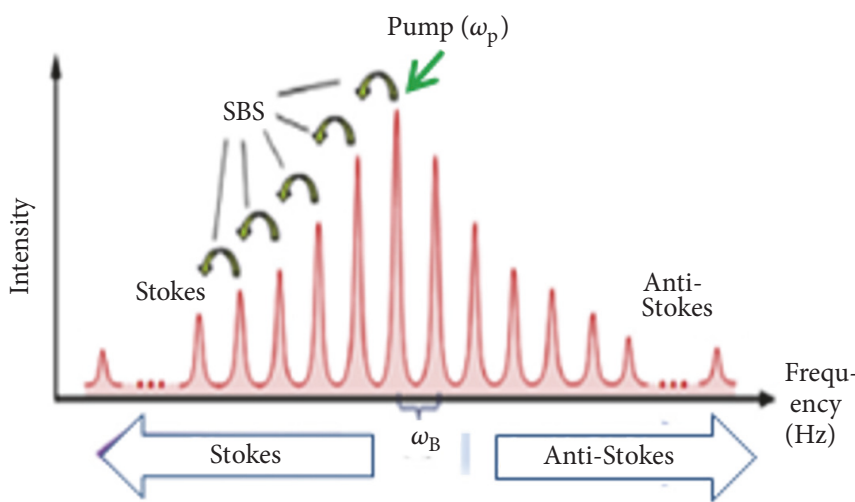

(b)

FIGURE 11: Principle of Brillouin frequency comb (BFC) generation [adapted from [5]].

5.11. Low Noise, Narrow Linewidth, and Frequency Combs. Low Noise: The SBS parametric process has intrinsically very low noise, since it does not initiate from spontaneous emission. It initiates from thermal vibrations, which represent the final limit of low noise.

SBS can produce the narrowest linewidths of any optical signal. Theoretical evaluation of SBS plus feedback from a resonator shows that the intrinsic laser linewidth is limited by thermal phonons to less than $1 \mathrm{~Hz}$. Such theories include forward, backward, and acoustic density waves interacting within a micro-resonator. Coupled-mode analysis with noise identifies fundamental parameters and their optimization for low noise SBS. Intrinsic laser linewidth is limited by thermal phonons $<\sim 1 \mathrm{~Hz}$, with pump noise reduced by stabilizing the pump laser onto a micro-cavity resonance which reduces pump noise. Any noise in the pump increases SBS laser noise beyond its fundamental limits.

Narrow Linewidth: SBS has been shown to produce a very narrow gain spectrum when SBS amplifiers are cascaded. Theoretically the gain linewidth of a SBS system decreases as the square root of the number of amplifiers. Experiments have shown that a $200 \mathrm{MHz}$ linewidth can be narrowed to $20 \mathrm{MHz}$.

A number of experiments have demonstrated both very narrow linewidths and very low signal-to-noise ratio. The lowest measurements of these parameters are in Brillouin lasers, particularly in erbium-doped fiber ring lasers. The systems can produce highly coherent SBS with $20 \mathrm{~dB}$ reduction of random intensity noise and frequency noise. However, stable operation also requires active stabilization. Locking the $\mathrm{GHz}$ resonance between the pump laser frequency and the Brillouin cavity mode offers the maximum stability.

Frequency Combs: A large number of publications have evaluated not only the narrow linewidth produced by cascading SBS in a feedback system, but the value of the resulting frequency combs. The principle of frequency comb generation is sketched in Figure 11. Comb generation requires feedback so that the laser pump can travel backward, through the acoustic wave created by Stokes generation. The alreadyoscillating density wave feeds the Brillouin shift back to the laser, enabling anti-Stokes as well as Stokes. As each of these lines becomes powerful, they are able to drive more and more orders of lines, each with a successive Brillouin shift. As shown in the figure, this provides a very broad comb of frequencies. The anti-Stokes wavelengths come from powerful backward-going laser pump interacting with the backward-traveling Brillouin-excited acoustic waves. The SBS process reflects the laser wave back into the forward direction, but adds the phonon energy to the forward-going laser photons, creating anti-Stokes.

A recent research paper provides just one example that shows how rapidly the field is progressing. Its design is shown in Figure 12. The originality here is that feedback in the main fiber cavity (i) is introduced by a Rayleigh-reflecting fiber. As backward Stokes progresses from the Brillouin gain loop through the optical circulator 2 , it is retroreflected by means of the Rayleigh fiber. This relies on stationary imperfections in the fiber causing reflection without any frequency shift. The subloop (ii) shown in Figure 11 enables efficient cascading, producing a Brillouin frequency comb of narrow frequencies [14].

The pump laser was erbium-doped $1550 \mathrm{~nm}$ fiber. The system consists of the main fiber cavity (i) and a fiber subloop (ii), which amplifies each backward Stokes wave through an erbium-doped fiber amplifier (EDFA). An optical coupler removes the signal from the loop through port 2. After polarization compensators, the signal enters the main cavity through optical circulator 1 and travels through the Brillouin gain fiber, which creates a Stokes signal that travels backward, as shown. Reaching optical circulator 2, the Stokes signal reflects backward toward the Brillouin gain fiber, as shown by the circulation arrows. Port \#1 represents the rear mirror for the Stokes laser. Many orders of Stokes emission simultaneously resonate in a coherent lasing fashion under the acoustic coupling of SBS in the presence of coherent Rayleigh scattered random feedback, which acts as an optical filter with the highest reflection coefficient for the lowest order longitudinal mode of each Stokes line, ensuring single longitudinal mode operation [27].

Frequency combs have been shown to have enumerable applications. Nobel prizes in 2005 for Jan Hall of NIST 


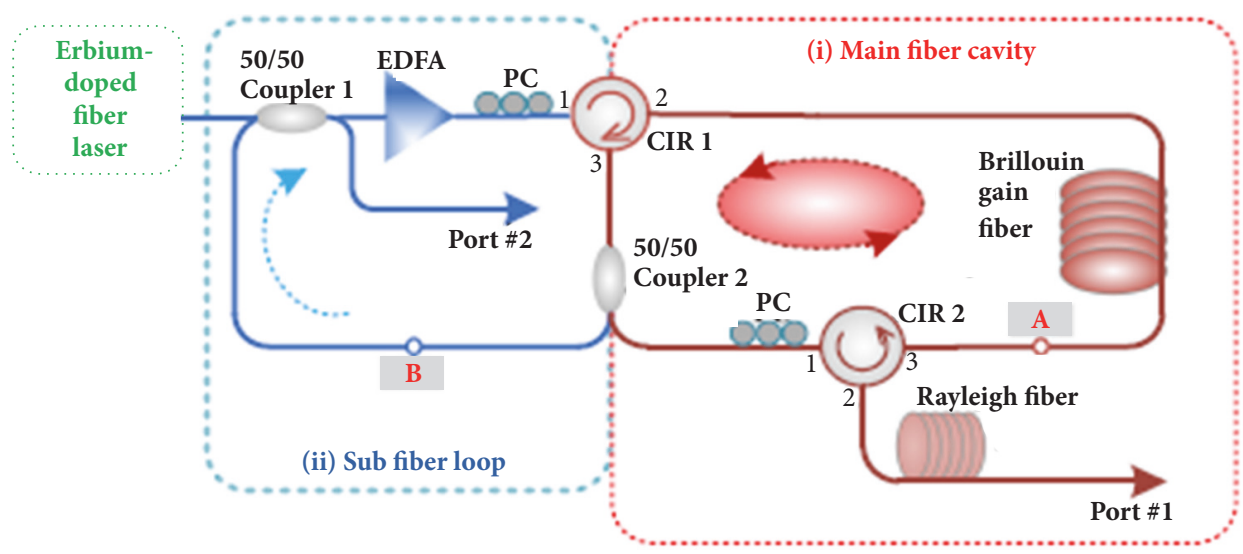

FIGURE 12: (i) Main fiber cavity: Optical circulator 1, Brillouin gain SM fiber, another circulator combining with Rayleigh feedback fiber (5$\mathrm{km}$ ) with continuously changing core diameter and dispersion for enhanced Rayleigh DFB. (ii) Fiber subloop: EDFA amplifies each backward Stokes wave [adapted from [14]].

and Ted Hansch of the Max Planck Institute were based on research with applications of frequency combs to precision spectroscopy and optical frequency metrology. I encourage you to study frequency combs by themselves to see all their exciting applications:

(i) WDM for fiber communications

(ii) Optical frequency synthesis by control of comb

(iii) Optical frequency metrology

(iv) Optical atomic clocks

(v) Precision spectroscopy

(vi) Microwave photonics

(vii) Sensing

(viii) Optical wavelength filter: erbium optical fiber oscillator and SBS amplification.

(ix) Stabilizing lasers at molecular absorption wavelengths.

5.12. Dynamic Brillouin Gratings. SBS imaging was discussed above, motivated by biomedical applications. Many of these applications involve creating density gratings within the material, called Brillouin dynamic gratings (BDG). These gratings can be made stationary when researchers pump with light beams from both sides. When a probe enters at the stronger pump end, it is reflected with a red-shift. When a probe enters on the weaker pump side, it is blueshifted. When the two pumps are equal, a standing acoustic wave is formed so that probe light is reflected without a frequency shift. The simplest process is to form these phonons by interference with ultra-short pulses with high enough intensity to cause substantial Bragg diffraction of the probe out of the monitored beam.

The intent is to measure dynamic properties of the acoustic grating to determine its magnitude and how long it lasts. In order to separate probe from pump, many systems polarize the probe perpendicular to pump. When experiments are performed in fibers, this requires the use of polarization maintaining fiber. As far as fiber sensors are concerned, BDG are shown to have enhanced spatial resolution and to separate strain and temperature. BDG do require, however, providing a pump at both ends of the fiber, which is not always possible.

The applications of BDG are numerous; they are often used by material scientists to investigate soft matter. You can create Bragg gratings by interfering two coherent laser beams, but BDG constitute an easier technique because it is a selfcoherent process; you do not need to guarantee two coherent laser beams.

One application for BDG is as an optical switch; the acoustic grating can turn on or off a Stokes beam, providing an optical flip-flop. If two such switches are used in tandem, an optical memory can be created. In one case the memory element was a long, weak grating controllable by phasemodulated pulses $300 \mathrm{ps}$ long [29]. Storage time was limited by nonuniformities to about $10 \mathrm{~ns}$.

In summary, BDG occur in a sample when SBS acoustic waves are generated from both sides due to electrostriction and the laser reflects off the moving grating with a Doppler shift. As SBS is typically a small nonlinearity, BDG require high intensity pulses, from Q-switched or mode-locked lasers, or long lengths in fibers or high $\mathrm{Q}$ resonators.

\section{Burgeoning Stimulated Brillouin Applications: Optophonics}

I have invented another new word: Optophonics. This is a shortening of optical-phononics. Some have introduced the term phononics, but that term is already used by solid state physicists. Photo-acoustics is also already used to define a well-developed field. This leaves photo-phononics as another possibility, but I find optophonics as the simplest word to describe this new set of applications and their underlying science, which involves mechanical interactions between phonons and photons.

6.1. Optophonic Integrated Circuits. Optophonics involves acoustic waves (phonons) interacting with light via SBS on 
integrated circuit (IC) platforms (as shown in Figure 13, with some possible applications listed). The concept is that light signals can be processed by means of acoustical waves created by the highly nonlinear SBS process. It is worth our being reminded that SBS has a higher gain coefficient than SRS and occurs in all materials. This implies that optophonics can have an exciting future. Because the SBS frequency shift is in the microwave regime, optophonics can also be used to synthesize and process microwave signals. New applications include on-chip narrow-linewidth lasers using SBS and phonon-based optical delay and signal processing.

The technology driver in optophonics is to reduce the size of the IC chip, so as to reach SBS threshold at reasonably low power levels. Optophonics will probably require high $\mathrm{Q}$ resonators (although some suggestions involve slow light in highly dispersive fibers). A partial list of application possibilities is shown in Table 1.

Photonic bandgap materials or photonic crystals can be used to increase nonlinearities and lower the required optical powers for operation. Alternatively, acoustic waves generated by transducers may be part of the system.

Researchers in SBS are making enormous progress in demonstrating signal processing of microwave signals by optophonics. An optophonic chip can be used for processing microwave signals in the following way. The microwave signal modulates an optical beam, which is processed on the chip. It is then, in turn, detected by a high-speed photodiode and the resulting electrical signal is again a microwave signal, which has been processed by the SBS interaction. This subject merits its own review paper and is not discussed in detail here, since there were 180 papers on the subject of "signal processing and SBS" in 2016 alone! By contrast, ten years earlier there were one-tenth as many papers per year.

Most of the interest in optophonics has been siliconbased; however, silicon has the distinct disadvantage of a weak nonlinear Brillouin coefficient. A possible alternative pursued in Australia is a hybrid technology that deposits $\mathrm{A}_{2} \mathrm{~S}_{3}$ surrounded by sputtered silica [29]. This provides waveguides in a material with a strong Brillouin coefficient.

An alternative approach uses a set of new concepts in mini, micro, and nanodevices that add new physics to electrostriction to increase the SBS nonlinearity and lower its threshold by orders of magnitude. The most exciting progress is being made in individual microdevices that can be eventually integrated into an optophonics chip, extending even to nanodevices.

6.2. SBS Can Enhance Other Nonlinear Processes. Because optical nonlinear processes tend to be weak, researchers not only use SBS directly, but may employ it to enhance other nonlinearities, particularly in optophonics where practical devices can use SBS to improve performance. One example will be called out here [30], but many others have been suggested. The idea is to use SBS to improve phase matching in Four-Wave Mixing (FWM), thereby allowing for larger interaction lengths and lower powers. With SBS, dynamic control of phase matching is possible. This control is useful in FWM wavelength conversion and works for both amplitude- and phase-modulated signals in communications
TABLE 1: Optophonic circuit applications.
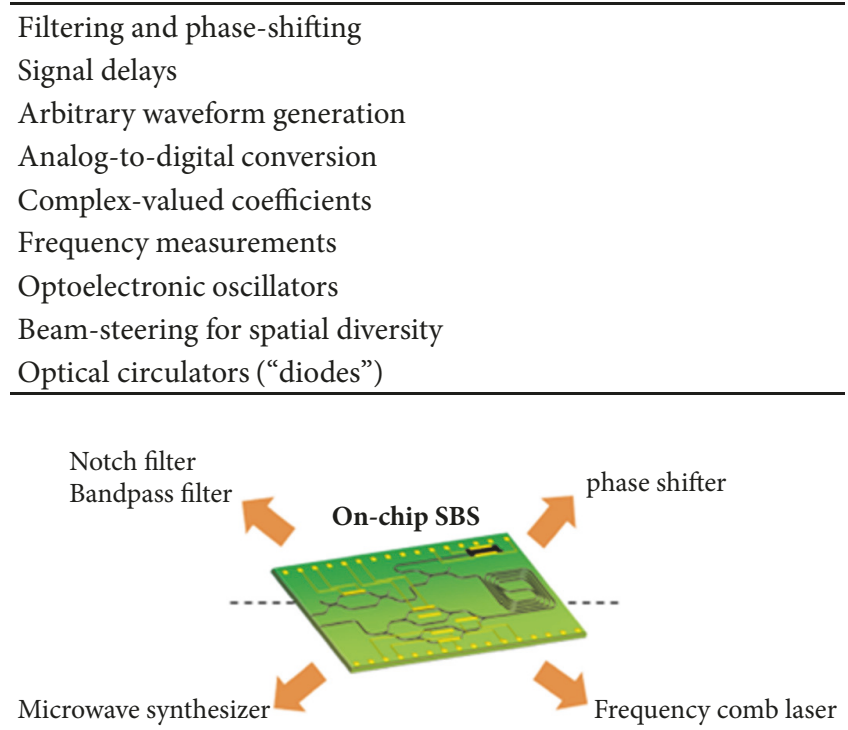

FIgURE 13: Concept for optophonics, a field which comes about from extending photonics by incorporating phonons as well as electronics into the field of integrated optics [adapted from [16]].

applications. SBS can offer a pure refractive change by introducing self-compensation of optical gain versus loss. This gives flexible control over conversion efficiency [30]. Experiments demonstrated an enhancement of the conversion efficiency in a communication system such that the $3-\mathrm{dB}$ conversion bandwidth for wavelength division multiplexing (WDM) increased by $41 \%$.

6.3. SBS in Microdevices. Demonstration of SBS in microdevices began with a waveguide at the surface of a $100 \mu \mathrm{m}$ silica ball [17]. The authors found maximum SBS gain when the acoustic wave was resonant around an equator of the silica ball [17], shown in Figure 14. They phase-matched Stokes and pump by using adjacent waveguide modes, both of which were resonated in the silica ball, as was the acoustic mode. They found that the light traveled in whispering gallery modes. Figure 14 shows a computer model of the acoustic wave intensity at $58 \mathrm{MHz}$ in their experiment, which illustrates the resonated pump and Stokes waves and the standing acoustic mechanical wave at the surface.

These ring resonators define for us two useful concepts: SBS-induced transparency and the use of forwardscattered SBS. The term SBS-induced transparency follows from the quantum atomic physics concept of self-induced transparency. A normally absorbing atomic system becomes transparent when light is powerful enough to drive half atoms into the excited state, removing absorption. For larger powers, the system might demonstrate gain. The same thing happens in SBS, only with acoustic phonons rather than atoms. SBS-induced transparency is possible only when the phonons have lifetimes longer than the photon lifetimes and the nonlinear medium can sustain sizeable acoustic vibrations. It requires forward-scattering SBS, for which the 


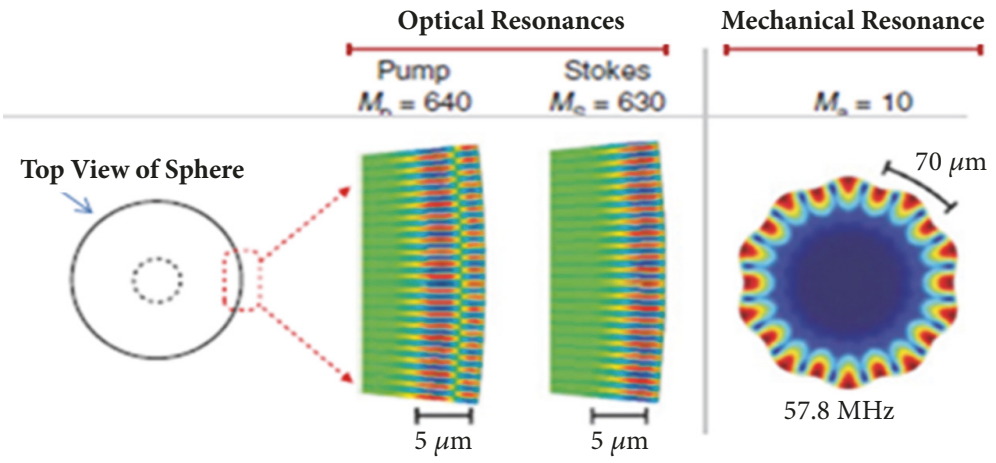

FIGURE 14: Simulation of interacting optical and mechanical modes at the surface of a spherical micro-device [adapted from [17]].

phonons typically have $\mathrm{MHz}$ frequencies, which means the acoustic waves are low-loss and remain for a long time. Their experiments have demonstrated SBS-induced transparency, stimulated optomechanical excitation of surface acoustic waves (SAW), and SBS cooling [17].

Figure 15 shows their theory and observation of SBSinduced-transparency for Stokes light, occurring in a very narrow frequency range, exactly on the Brillouin resonance. The top trace shows traditional SBS theory for short-lived phonons: gain for Stokes, loss for anti-Stokes. The second graph shows what occurs when phonons are long-lived: selfinduced transparency exactly at the anti-Stokes frequency. Apparent time reversal asymmetry can be seen in the results because if the probe enters backward, there is no impact at the Stokes frequency. However, for the forward-traveling probe, SBS-induced optical transparency does occur around the anti-Stokes frequency. In fact, however, reversing only the probe direction is not true time reversal because the pump does not change direction, which full time reversal would require. Nonetheless this geometry represents a useful optical circulator for the probe [18]. Similar results were obtained, essentially simultaneously, by [31].

One geometry for coupling into micro-resonators like the silica ball is shown in Figure 16, showing its design for use in optical signal processing. The control signal is brought in on a fiber at frequency $\omega_{c}$ and the probe is varied in frequency to find a resonance for both the resonator and the Brillouin line. Ideally the input control signal is resonated also. A tapered fiber optimizes the coupling. Brillouin ScatteringInduced Transmission (BSIT) has been measured in such resonators, including the forward SBS interaction. The frequency shifts are smaller in the forward direction so that signal processing at microwave frequencies is one possible application.

6.4. Ultra-High Q Micro-Lasers. Exciting new work in micro and nano structures shows that, with very high Q's ( $10^{8}$ ), thresholds can be exceeded for SBS lasers on-chip when excited by moderate pump laser power. A planar ring resonator $1 \mathrm{~mm}$ in diameter was made at Caltech with a wedge-shaped edge around the ring, in order to control the waveguide mode profile to match the input fiber coupler. To measure the intrinsic Q-factor, the devices were coupled to a SMF-28 optical fiber taper. Spectral lineshape data were obtained by tuning an external cavity semiconductor laser across the resonance while monitoring transmission on an oscilloscope. Figure 17(a) shows a microphotograph of the ring resonator. Figure 17(b) shows the spectrum coupled out of resonator, both the Stokes line and a bit of scattered laser light. Apparently these resonators operate effectively with whispering gallery modes.

Continuing work at Caltech has aimed to isolate the cavity acoustically by providing a pedestal for the disk laser. This geometry has been used in a possible application as a gyro. Gyros measure rotation by the Sagnac-induced frequency shift. Here the measurement is of the change in resonant wavelength when the ring is rotated. Their data showed forty times more sensitivity than other ring resonators. The device was a high Q microresonator, $18 \mathrm{~mm}$ in diameter, fabricated in silica deposited on a silicon chip. Counter-propagating Brillouin Stokes lasers excited in this resonator are coupled into a fast photodetector that measures the microwave beat note at $10.8 \mathrm{GHz}$ between the $2^{\text {nd }}$ and $3^{\text {rd }}$ Stokes waves. Sign of the Brillouin change in beat note indicates rotation direction. [32]

6.5. SBS in Nanoscale Waveguides. Linear micro-waveguides are made more effective for SBS with proper design in which the acoustic waves can also be resonated. As the waveguides shrink, radiation pressure on the light becomes large and adds to electrostriction. This realization has led researchers to push beyond microscale to nanoscale waveguides.

The cross-section of one experimental nanoscale waveguide fabricated in silicon is shown in Figure 18. Experiments demonstrated that forward Brillion Stokes gain was 1000 times larger than backward. Enhanced emission of phonons was observed, as predicted by Professor Rakich from Yale [33]. Brillouin phonons in these nanowaveguides had frequencies from 1 to $18 \mathrm{GHz}$. Experiments showed that radiation pressures, produced by subwavelength modal confinement, yield enhancement of the Brillouin nonlinearity well beyond those of material nonlinearity alone.

Further studies of nanoscale waveguides have confirmed that, in highly confined waveguides, boundary forces due to radiation pressure and electrostriction increase the Brillouin gain. Simultaneous confinement of optical and acoustic fields 

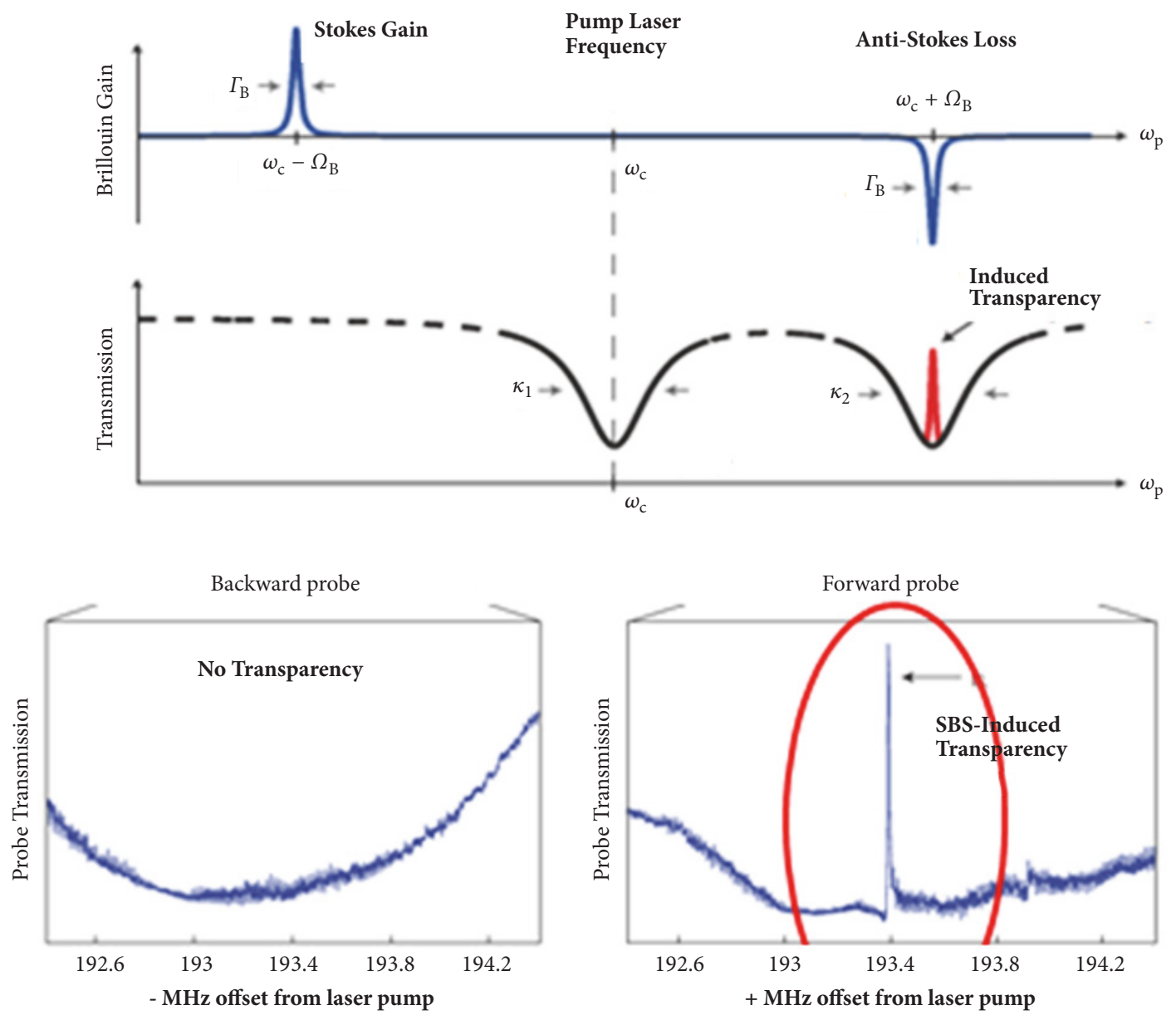

FIGURE 15: Demonstration of SBS-induced transparency in a micro-resonator. Top: Theory of Brillouin signal versus probe frequency; a probe at Stokes wavelength experiences gain, while loss is predicted for a probe signal at the anti-Stokes frequency. At high excitation levels, induced transparency is expected exactly on the Brillouin resonance. Bottom: when the phonon is long-lived, loss is observed at the Stokes frequency, while a probe at the anti-Stokes frequency experiences the predicted SBS-induced transparency [adapted from [18]].

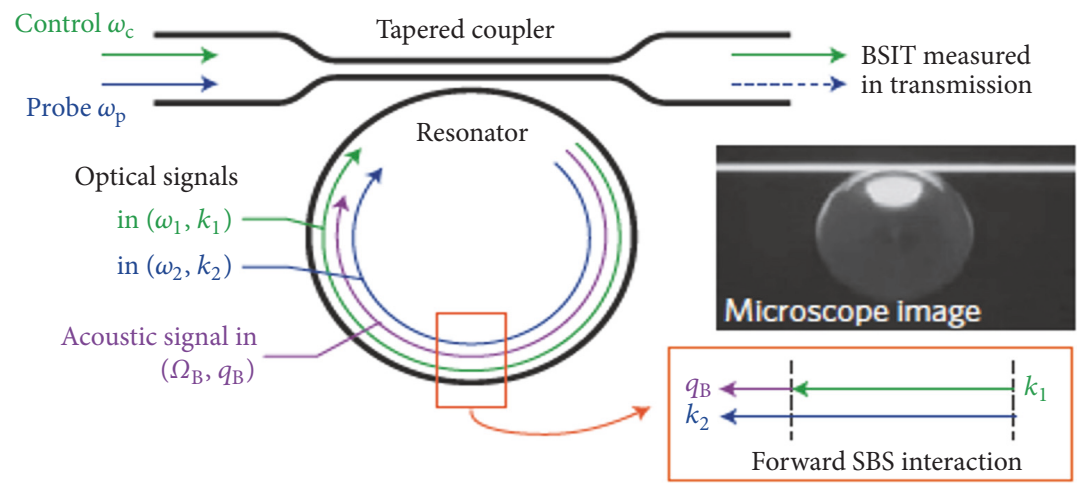

FIGURE 16: Geometry of tapered fiber coupler to the transparent sphere, plus a photograph of the coupler [adapted from [18]].

near a free surface or interface results in ultra-low SBS thresholds. An alternative, of course, is whispering gallery nanoresonators (WGRs) with ultra-high Q that have created gain enhancements as high as $10^{8}$ times [21]; a SEM image of such a resonator is shown in Figure 19. Clearly this is an important direction for the future of SBS.
Other research has offered similar arguments and points out that acoustic waves can leak out of their resonator unless the mounting to the substrate is as delicate as possible. The SEM images presented to date and modelling results are impressive and suggest that there remain huge possibilities for creative designs in the future [22]. In fact, there have recently 


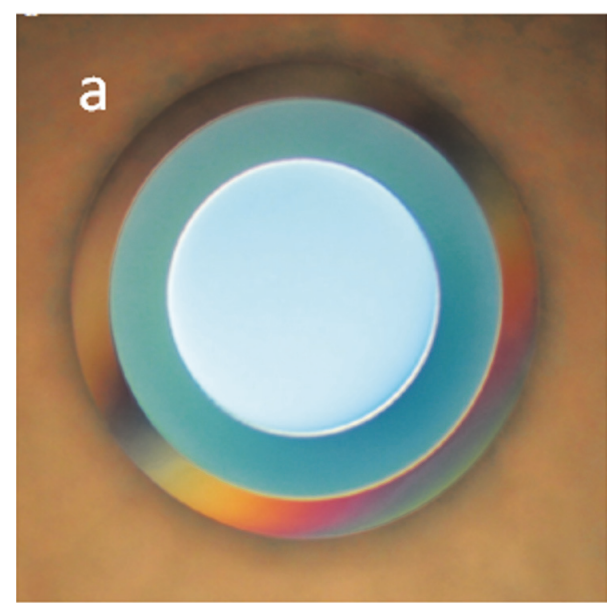

(a)

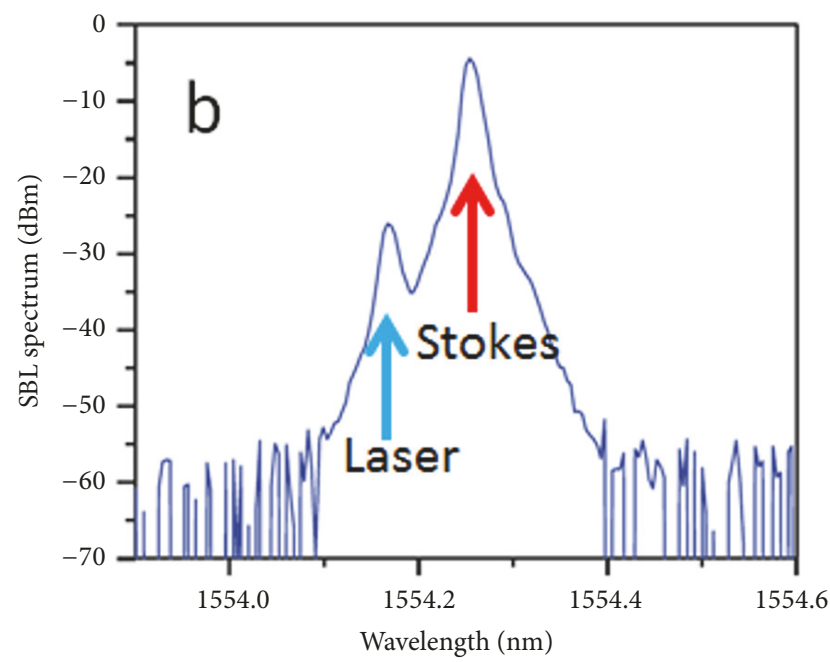

(b)

FIGURE 17: (a) Ring resonator for SBS laser, $1 \mathrm{~mm}$ in diameter; (b) Brillouin scattering spectrum [adapted from [20]].

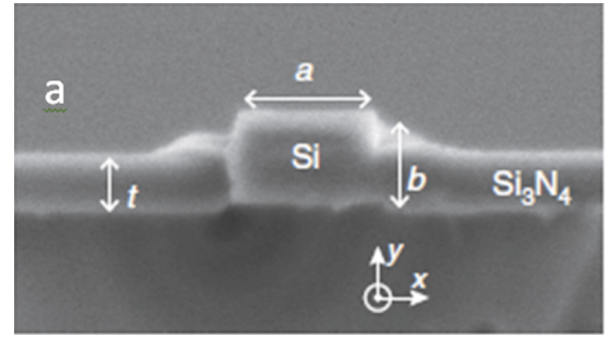

(a)

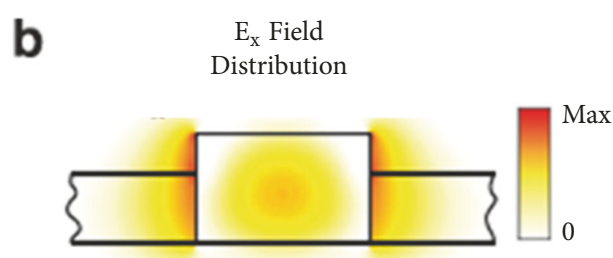

(b)

FIGURE 18: (a) Cross-section of a linear nanowaveguide of width $313 \mathrm{~nm}$ and height $194 \mathrm{~nm}$; (b) computer model of the field distribution of its guided wave [adapted from [21]].

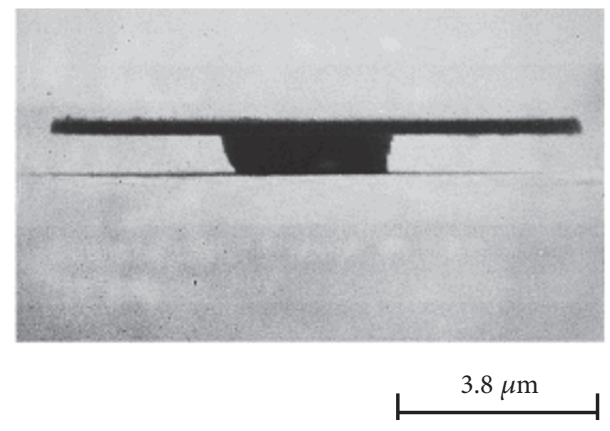

FIGURE 19: A scanning electron microscopy image of the side view of an Er-doped $\mathrm{GaP}$ microdisk resonator with $10 \mu \mathrm{m}$ diameter [adapted from [22]].

been a large number of papers on the topic of SBS in high Q resonators.

\section{Conclusion}

One recent paper in Physical Review Letters [34] caught my eye. Its title intrigued me: "Optical Force Enhancement using Imaginary Vector Potential for Photons." The concept of "imaginary vector potential" already frightened me, but the paper also introduced the concept of "gauge materials," which frightened me even more. The paper suggests that "Gauge materials should emulate imaginary vector potentials." It suggests implementing "complex-valued vector potentials with non-Hermitian waveguide cores." This is clearly outside my present research capabilities within electrical engineering, but promises whole new ways of thinking about nonlinearities. I am glad that I am retired and I do not need to expand my understanding to include these new concepts. However, it did inspire me to see that this paper, from Harvard, included two women among its four authors. Times have changed since I was the only woman graduate student in Physics at MIT! The future offers huge potential research directions in nonlinear optics with SBS. Which direction will provide the most applications in the next 50 years? It is an exciting time for all who want to become optical scientists and engineers to think about the future of nonlinear optics.

\section{Disclosure}

This paper is an outgrowth of a talk I gave at the Conference on Lasers and Electro-Optics (CLEO, May 2018 in Long 
Beach CA, USA) entitled "Stimulated Brillouin Scattering: Then and Now."

\section{Conflicts of Interest}

The author declares that there are no conflicts of interest regarding the publication of this paper.

\section{Acknowledgments}

The author acknowledges the tremendous number of researchers who have worked in this field over many years. Without their publishing their research in the open literature, this paper could not have been published. As an emerita professor, the author acknowledges Dartmouth College, its library, and other resources which were made available to complete this review.

\section{References}

[1] E. Garmire, F. Pandarese, and C. H. Townes, "Coherently driven molecular vibrations and light modulation," Physical Review Letters, vol. 11, no. 4, pp. 160-163, 1963.

[2] R. Y. Chiao, E. Garmire, and C. H. Townes, "Raman and Phonon Masers," in Proceedings of the 1963 International School of Physics Enrico Fermi, Course XVII, P. Miles, Ed., Academic Press, New York, USA, 1968.

[3] R. Y. Chiao, C. H. Townes, and B. P. Stoicheff, "Stimulated Brillouin scattering and generation of intense hypersonic waves," Physical Review Letters, vol. 12, no. 21, pp. 592-595, 1964.

[4] E. Garmire and C. H. Townes, "Stimulated brillouin scattering in liquids," Applied Physics Letters, vol. 5, no. 4, pp. 84-86, 1964.

[5] Z. Bai, H. Yuan, Z. Liu et al., "Stimulated Brillouin scattering materials, experimental design and applications: A review," Optical Materials, vol. 75, pp. 626-629, 2018.

[6] D.-P. Liu and Y. Hu, "Novel design of laser with SBS phase conjugation mirror," International Journal of Infrared and Millimeter Waves, vol. 26, no. 1, pp. 125-131, 2005.

[7] G. Scarcelli and S. H. Yun, "Confocal Brillouin microscopy for three-dimensional mechanical imaging," Nature Photonics, vol. 2, no. 1, pp. 39-43, 2008.

[8] C. H. Townes, E. Garmire, R. Chiao, and B. Stoicheff, HighFrequency Phonon Generating Apparatus And Method, U.S. Patent \# 3,469,107, 1969, https://patents.google.com/patent/ US3469107.

[9] C. W. Ballmann, Z. Meng, A. J. Traverso, M. O. Scully, and V. V. Yakovlev, "Impulsive brillouin microscopy," Optica, vol. 4, no. 1, pp. 124-128, 2017.

[10] C. W. Ballmann, J. V. Thompson, A. J. Traverso, Z. Meng, M. O. Scully, and V. V. Yakovlev, "Stimulated Brillouin Scattering Microscopic Imaging," Scientific Reports, vol. 5, no. 1, Article ID 18139, 2015.

[11] Z. Bai, Y. Wang, Z. Lu et al., "High Compact, High Quality Single Longitudinal Mode Hundred Picoseconds Laser Based on Stimulated Brillouin Scattering Pulse Compression," Applied Sciences, vol. 6, no. 1, p. 29, 2016.

[12] J. P. Cahill, O. Okusaga, and J. O. White, "Suppression of stimulated Brillouin scattering in high power, low phase noise RF-photonic links," in Proceedings of the 2012 66th IEEE
International Frequency Control Symposium, IFCS 2012, pp. 677680, USA, May 2012.

[13] Z. Zhao, Y. Dang, M. Tang et al., "Spatial-division multiplexed Brillouin distributed sensing based on a heterogeneous multicore fiber," Optics Letters, vol. 42, no. 1, pp. 171-174, 2017.

[14] L. Zhang, Y. Xu, S. Gao et al., "Multi-wavelength coherent Brillouin random fiber laser with ultrahigh optical SNR," IEEE Journal of Selected Topics in Quantum Electronics, vol. 24, no. 3, 2018.

[15] B. Ya. Zeldovich, V. I. Popovichwc, V. V. Ragulskiy, and F. S. Faizullov, "Connection between the wave fronts of the reflected and exciting light in stimulated Mandelshtam-Brillouin scattering," JETP Letters-USSR, vol. 15, no. 3, pp. 109-111, 1972.

[16] B. Morrison, A. C. Bedoya, G. Ren et al., "Compact Brillouin devices through hybrid integration on silicon," Optica, vol. 4, no. 8, pp. 847-854, 2017.

[17] G. Bahl, J. Zehnpfennig, M. Tomes, and T. Carmon, "Stimulated optomechanical excitation of surface acoustic waves in a microdevice," Nature Communications, vol. 2, no. 1, 2011.

[18] J. H. Kim, M. C. Kuzyk, K. Han, H. Wang, and G. Bahl, "Nonreciprocal Brillouin scattering induced transparency," Nature Physics, vol. 11, no. 3, pp. 275-280, 2015.

[19] S. Park, S. Cha, J. Oh et al., "Coherent beam combination using self-phase locked stimulated Brillouin scattering phase conjugate mirrors with a rotating wedge for high power laser generation," Optics Express, vol. 24, no. 8, pp. 8641-8646, 2016.

[20] H. Lee, T. Chen, J. Li et al., "Chemically etched ultrahigh-Q wedge-resonator on a silicon chip," Nature Photonics, vol. 6, no. 6, pp. 369-373, 2012.

[21] H. Shin, W. Qiu, R. Jarecki et al., "Tailorable SBS in nanoscale silicon waveguides," Nature Communications, vol. 4, p. 1944, 2013.

[22] R. Van Laer, B. Kuyken, D. Van Thourhout, and R. Baets, "Interaction between light and highly confined hypersound in a silicon photonic nanowire," Nature Photonics, vol. 9, no. 3, pp. 199-203, 2015.

[23] E. Edrei and G. Scarcelli, "Brillouin micro-spectroscopy through aberrations via sensorless adaptive optics," Applied Physics Letters, vol. 112, no. 16, p. 163701, 2018.

[24] I. Remer and A. Bilenca, "High-speed stimulated Brillouin scattering spectroscopy at $780 \mathrm{~nm}$," APL Photonics, vol. 1, no. 6, p. 061301, 2016.

[25] C. Feng, X. Xu, and J.-C. Diels, "High-energy sub-phonon lifetime pulse compression by stimulated Brillouin scattering in liquids," Optics Express, vol. 25, no. 11, pp. 12421-12434, 2017.

[26] G. Yang, X. Fan, and Z. He, "Strain dynamic range enlargement of slope-assisted BOTDA by using brillouin phase-gain ratio," Journal of Lightwave Technology, vol. 35, no. 20, pp. 4451-4458, 2017.

[27] L. Wang, N. Guo, C. Jin et al., "Coherent BOTDA using phaseand polarization-diversity heterodyne detection and embedded digital signal processing," IEEE Sensors Journal, vol. 17, no. 12, pp. 3728-3734, 2017.

[28] Yongkang Dong, Jiang Ba, Taofei Dexin et al., "High-SpatialResolution Fast BOTDA for Dynamic Strain Measurement Based on Differential Double-Pulse and Second-Order Sideband of Modulation," IEEE Photonics Journal, vol. 5, no. 3, Article ID 2600407, 2013.

[29] M. A. Soto, A. Denisov, X. Angulo-Vinuesa, S. Martin-Lopez, L. Thévenaz, and M. Gonzalez-Herraez, "All-optical flip-flops based on dynamic Brillouin gratings in fibers," Optics Letters, vol. 42, no. 13, pp. 2539-2541, 2017. 
[30] L. Wang and C. Shu, "Dynamic control of phase matching in four-wave mixing wavelength conversion of amplitude-and phase-modulated signals," Journal of Lightwave Technology, vol. 31, no. 9, pp. 1468-1474, 2013.

[31] C. H. Dong, Z. Shen, C. L. Zou, Y. Zhang, W. Fu, and G. Guo, "Brillouin-scattering-induced transparency and non-reciprocal light storage," Nature Communications, vol. 6, no. 1, Article ID 7193, 2015.

[32] J. Li, M.-G. Suh, and K. Vahala, "Microresonator Brillouin gyroscope," Optica, vol. 4, no. 3, pp. 346-348, 2017.

[33] P. T. Rakich, C. Reinke, R. Camacho, P. Davids, and Z. Wang, "Giant enhancement of stimulated Brillouin scattering in the subwavelength limit," Physical Review X, vol. 2, no. 1, p. 011008, 2012.

[34] L. Descheemaeker, V. Ginis, S. Viaene, and P. Tassin, "Optical Force Enhancement Using an Imaginary Vector Potential for Photons," Physical Review Letters, vol. 119, no. 13, 2017. 

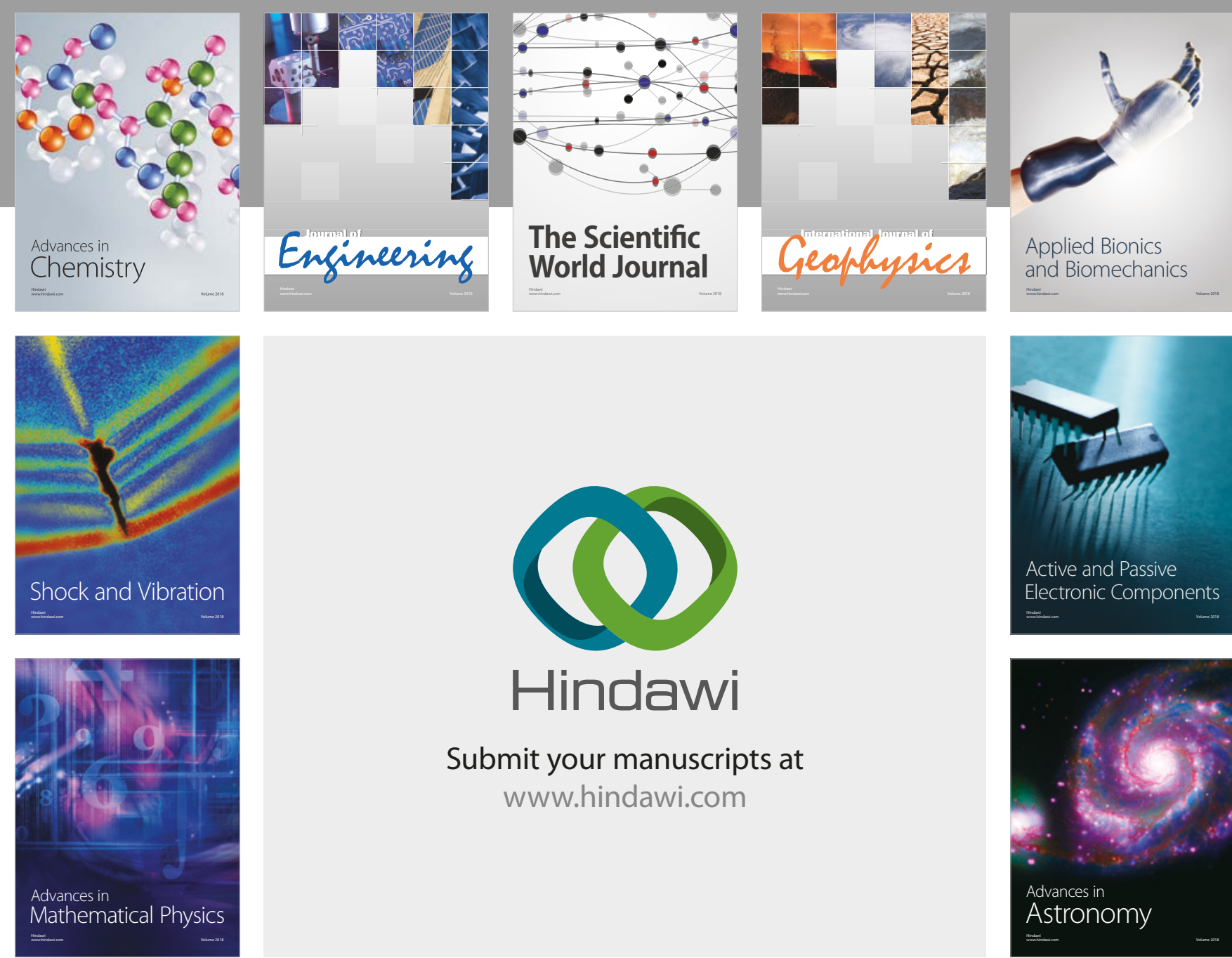

Submit your manuscripts at

www.hindawi.com

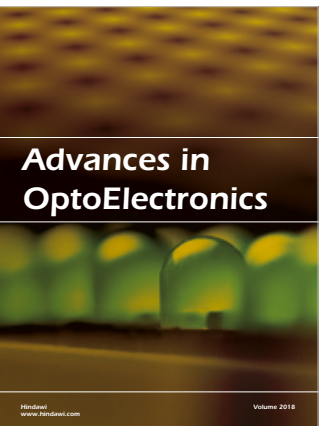

\section{Rotcting Machinery}
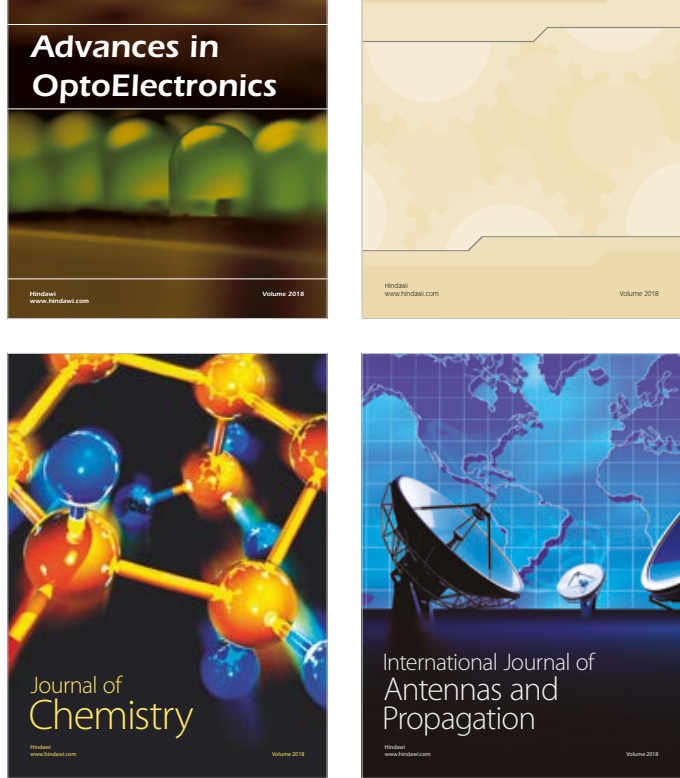

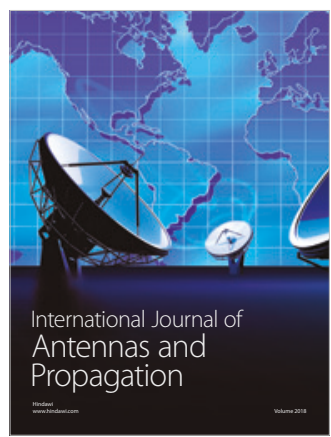

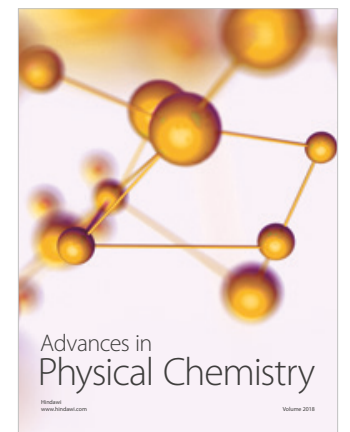

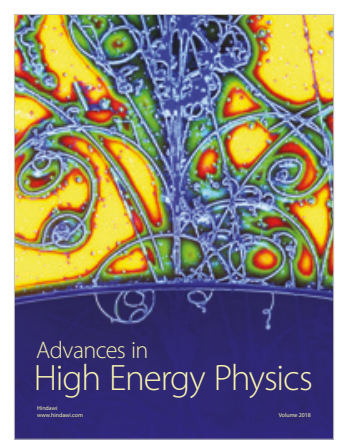

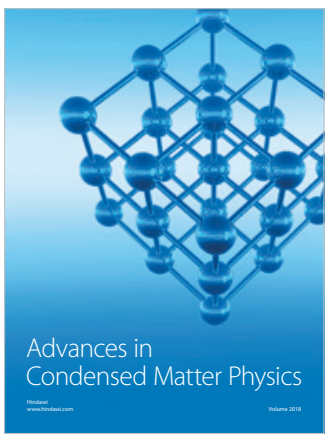

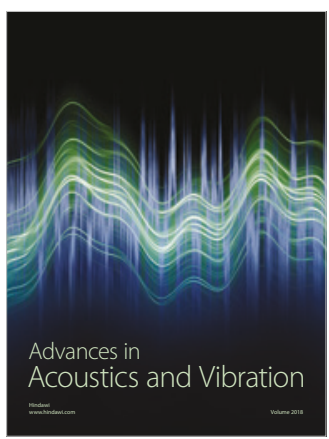

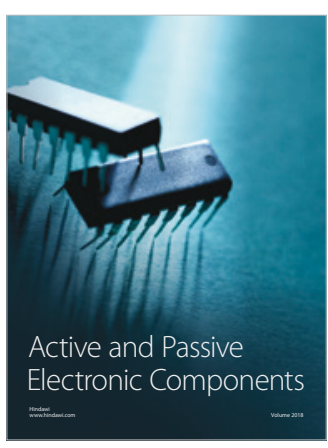
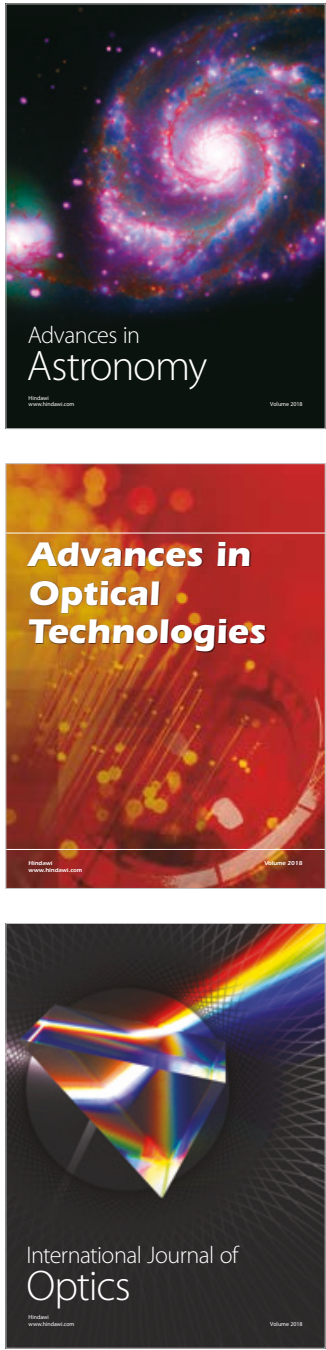